\title{
Fundamental Configurations in Mo IV Spectrum
}

This article has been downloaded from IOPscience. Please scroll down to see the full text article. 1987 Phys. Scr. 35819

(http://iopscience.iop.org/1402-4896/35/6/010)

View the table of contents for this issue, or go to the journal homepage for more

Download details:

IP Address: 161.111.22.141

The article was downloaded on 26/03/2013 at 09:54

Please note that terms and conditions apply. 


\title{
Fundamental Configurations in Mo IV Spectrum
}

\author{
M. T. Fernandez, I. Cabeza, L. Iglesias, O. Garcia-Riquelme and F. R. Rico
}

Instituto de Optica, Serrano 121, Madrid, Spain

and

\section{Kaufman}

Atomic and Plasma Radiation Division, National Bureau of Standards, Gaithersburg, MD 20899, U.S.A.

Received November 28, 1986; accepted January 30, 1987

\begin{abstract}
The spectrum of Mo IV was produced in a sliding-spark discharge and photographed with the $10.7 \mathrm{~m}$ normal-incidence vacuum spectrograph at the NBS in the $600-3200 \AA$ spectral region. All 35 levels of the $4 d^{3}$ and $4 d^{2} 5 s$ even configurations and all 45 of the levels of the $4 d^{2} 5 p$ odd configuration have been established from the 514 line classifications in the $800-3150 \AA$ region. Parametric calculations have been made for the even level systems with configuration interaction and for the odd configuration.
\end{abstract}

\section{Introduction}

The fourth spectrum of molybdenum was analyzed originally by A. Y. Eliason [1] in 1933. He published 8 levels of the even configurations $4 d^{3}$ and $4 d^{2} 5 s$ and 12 levels of the odd configuration $4 d^{2} 5 p$. We have studied these Mo IV fundamental configurations from new observations and established all of the 35 even and all of the 45 odd levels. We have confirmed Eliason's levels although the energy values have been slightly changed due to the better accuracy of the new observations.

\section{Experimental method}

The spectrum of molybdenum was photographed in the wavelength region $600-3200 \AA$ using the NBS $10.7 \mathrm{~m}$ normalincidence vacuum spectrograph equipped with a $12001 / \mathrm{mm}$ grating and having a plate factor of $0.77 \AA / \mathrm{mm}$. A slidingspark light source was used [2]. Discrimination of the ionization stages was made possible using peak current discharges between 30 and $500 \mathrm{~A}$, producing a good separation of Mo III, Mo IV and Mo V lines. In some cases, it was necessary to introduce helium at approximately $20 \mathrm{Torr}$ in order to maintain the discharge. The spectra were recorded on Kodak SWR plates in the wavelength region below $2500 \AA$ and on $103 \mathrm{a} 0$ plates above $2500 \AA$. The intensity distribution along the spectral lines and the behaviour of the line intensity at different peak currents were used to attribute lines to the fourth spectrum.

The spectrograms were measured on the Abbe comparator at the Instituto de Optica in Madrid. The intensity of the lines are relative visual estimates of the photographic blackening. Almost all of the lines were measured at least three times. Reference lines of $\mathrm{Cu}$ I, Cu II, Ge I, Ge II, Ar I, Ar II [3] and impurity lines of $\mathrm{O}, \mathrm{C}$ and $\mathrm{Si}$ [4] were used to calculate the wavelengths of the measured lines. Their uncertainty is estimated to be $0.005 \AA$.

The Mo $\mathrm{V}$ lines on our plates have been used in " $\mathrm{A}$ revision of the analysis of the fifth spectrum of Molybdenum
(Mo V)" [5]. The Mo III lines are being used in a revision and extension of the $\mathrm{Mo}^{++}$ion spectrum now in progress.

\section{Analysis}

We first checked the 8 energy levels of $4 d^{3}{ }^{4} F$ and $4 d^{2}\left({ }^{3} F\right) 5 s^{4} F$ from the even configurations and the 12 odd levels of the $4 d^{2}\left({ }^{3} F\right) 5 p{ }^{4} G,{ }^{4} F$ and ${ }^{4} D$ terms, which were reported by Eliason [1]. All of his levels were confirmed, although the values have been slightly modified due to the better accuracy of our observations.

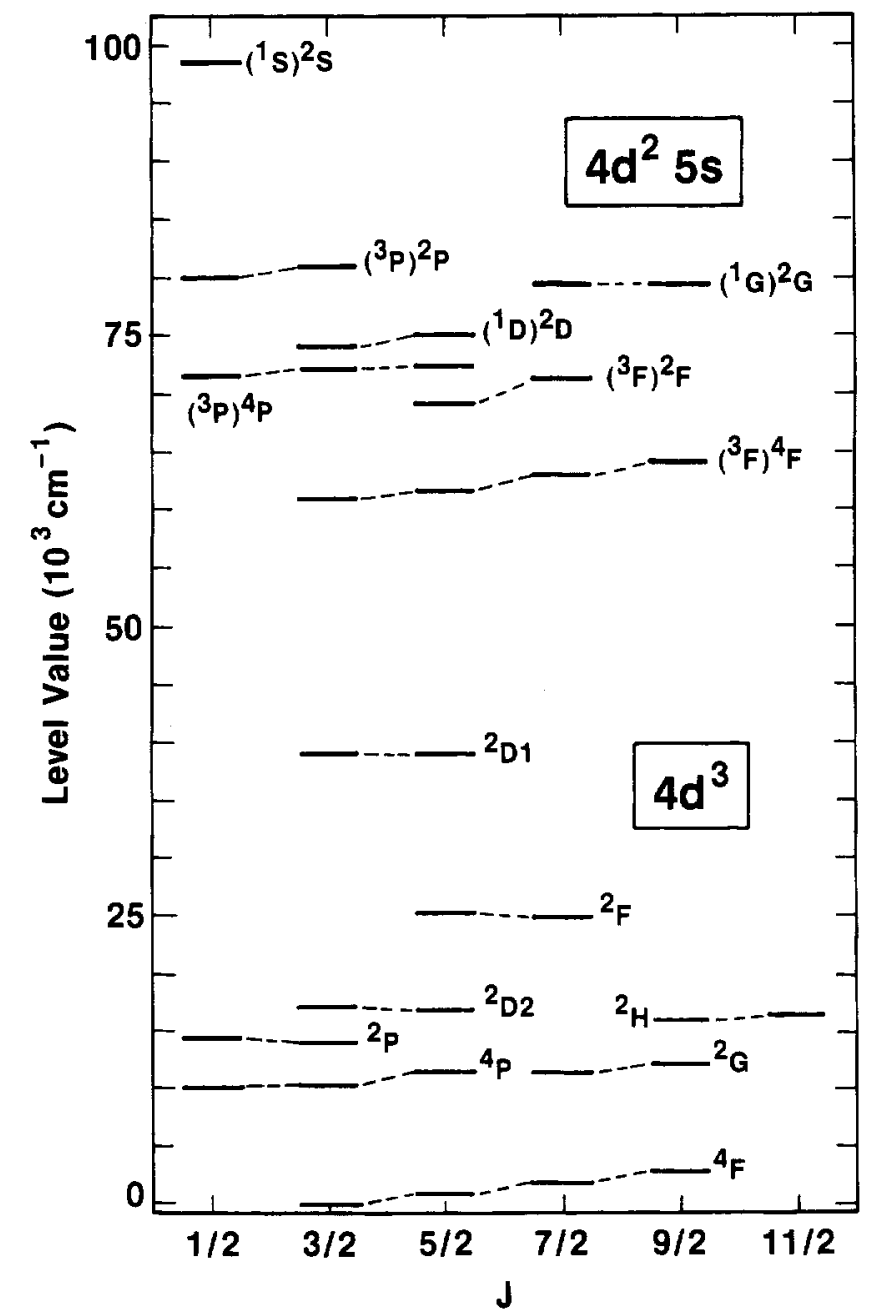

Fig. 1. Energy levels of the $4 d^{3}$ and $4 d^{2} 5 s$ even configurations of triply ionized molybdenum. The levels are connected to form $L S$ terms. 
The wavenumber differences between these known levels were used with the observed lines to find new levels. The level structure, as predicted by parametric calculations, and the isoelectronic spectra, $\mathrm{Zr} \mathrm{II} \mathrm{[6]} \mathrm{and} \mathrm{Nb} \mathrm{III} \mathrm{[7],} \mathrm{were} \mathrm{helpful} \mathrm{in}$ the search. Final energy level values were obtained using an iterative least-squares fitting of the levels to the observed classified lines.

A total of 508 lines were classified as belonging to the $4 d^{3}-4 d^{2} 5 p$ and $4 d^{2} 5 s-4 d^{2} 5 p$ transition arrays of Mo IV. Six of these are doubly classified. Table I contains the wavelength, intensity, wavenumber and classification of each observed line, as well as the difference, $O-C$, between the observed and calculated wavelength. The $4 d^{3}-4 d^{2} 5 p$ transition array falls in the $800-1400 \AA$ region and that of the $4 d^{5} 5 s-4 d^{2} 5 p$ appears in the $1200-3130 \AA$ region.

All of the 35 levels of the even configurations, $4 d^{3}$ and $4 d^{2} 5 s$ are now known. A least squares fit of the radial coefficients to the observed energy levels, including configuration interaction, was made for these configurations.

We have found all 45 levels of the $4 d^{2} 5 p$ odd configuration. Levels of the $4 d 5 s 5 p$ configuration are not known but there is probably configuration interaction between levels of these two odd configurations. Therefore, the standard deviation of the least squares fit of the parameters to the levels of $4 d^{2} 5 p$ was larger than that for the even configurations.

Tables II and III give the relevant information about the even and odd levels, respectively. Included for each level in the tables are the configuration, term, $J$ value, the level value and its uncertainty, and the number of observed transitions to or from the level. The tables also include the difference between the observed level value and that calculated with the least-squares fit parameters, $O-C$, and the leading eigenvector percentage(s) in $L S$ coupling.

The levels of the even configurations, $4 d^{3}$ and $4 d^{2} 5 s$, have an average purity of $90 \%$ in the $L S$ coupling scheme. However, the $J=3 / 2$ levels of $4 d^{3}$ at 14175 and $17107 \mathrm{~cm}^{-1}$ are quite mixed as can be seen in Table II. Figure 1 is a graphical view of the level structure of the even configurations. The levels are connected to show the $L S$ terms. The levels of the $4 d^{2} 5 p$ odd configuration have an average $L S$ purity of $70 \%$. Eight of these levels have leading $L S$ percentage compositions of less than $50 \%$. However only the level at $122808 \mathrm{~cm}^{-1}$, designated as $4 d^{2}\left({ }^{1} D\right) 5 p^{2} P_{3: 2}$, is not named according to its largest eigenvector component. This leads to no ambiguity of the level names. Figure 2 shows the level structure of this configuration.

Table IV gives the parameters derived in the least-squares

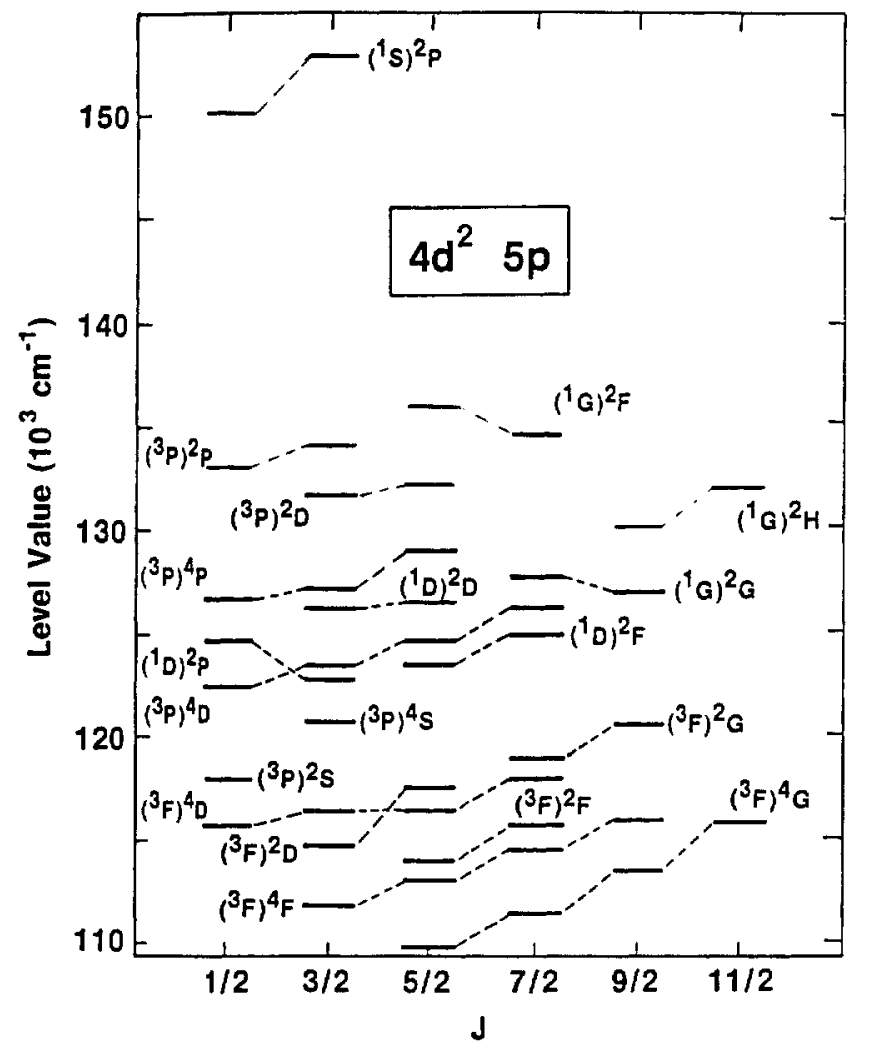

Fig. 2. Energy levels of the $4 d^{2} 5 p$ odd configuration of triply ionized molybdenum. The levels are connected to form $L S$ terms.

fit mentioned above. The standard deviation for these even configurations was $30 \mathrm{~cm}^{-1}$; that for the $4 d^{2} 5 p$ was $141 \mathrm{~cm}^{-1}$. The table also includes the relativistic Hartree-Fock values derived from the computer program of R. D. Cowan [8] as well as the ratio of the fitted to $a b$ initio values.

\section{References}

1. Eliason, A. Y., Phys. Rev, 43, 745 (1933).

2. Sugar, J., J, Opt. Soc. Am. 53, 831 (1963).

3. Kaufman, V. and Edlén, B., J. Phys. Chem. Ref. Data 3, 825 (1974).

4. Kelly, R. L. and Palumbo, L. J., Atomic and Ionic Emission Lines Below 2000 A - Hydrogen Through Krypton, NRL report 7599 (1973).

5. Cabeza, I., Meijer, F. G. and Iglesias, L., Phys. Scripta 34, 223 (1986).

6. Moore, C. E., U.S. Nat. Bur. Stnd, Circ. 467. Atomic Energy Levels, Vol. II, 209 (1952).

7. Iglesias, L., J. Opt. Soc. Am. 45, 856 (1955).

8. Cowan, R. D. and Griffin, D. C., J. Opt. Soc. Am. 66, 1010 (1976). 
Table I. Classified lines of Mo IV

\begin{tabular}{|c|c|c|c|c|c|c|c|c|c|}
\hline $\begin{array}{l}\text { Wavelength } \\
(\AA)\end{array}$ & Int. & $\begin{array}{l}\text { Wavenumber } \\
\left(\mathrm{cm}^{-1}\right)\end{array}$ & $O-C(\AA)$ & $\begin{array}{l}\text { Classification } \\
4 d^{3}, 4 d^{2}() 5 s-4 d^{2}() 5 p\end{array}$ & $\begin{array}{l}\text { Wavelength } \\
(\AA)\end{array}$ & Int. & $\begin{array}{l}\text { Wavenumber } \\
\left(\mathrm{cm}^{-1}\right)\end{array}$ & $O-C(\AA)$ & $\begin{array}{l}\text { Classification } \\
4 d^{3}, 4 d^{2}() 5 s-4 d^{2}() 5 p\end{array}$ \\
\hline 3137.700 & 2 & 31861.24 & -0.002 & $\left({ }^{3} P\right)^{2} P_{1 / 2}-\left({ }^{3} F\right)^{4} F_{3 / 2}^{0}$ & 2060.398 & 500 & 48518.79 & 0.007 & $\left({ }^{3} F\right)^{4} F_{3 / 2}-\left({ }^{3} F\right)^{4} G_{5 / 2}^{0}$ \\
\hline 3035.604 & 2 & 33932.78 & -0.001 & $\left({ }^{3} P\right)^{2} P_{3,2}-\left({ }^{3} F\right)^{2} F_{5 / 2}^{0}$ & 2059.704 & 300 & 48535.14 & 0.005 & $\left({ }^{\mathrm{l}} G\right)^{2} G_{7 / 2}^{3 / 2}-\left({ }^{1} G\right)^{2} G_{7 / 2}^{0}$ \\
\hline 2970.363 & 1 & 33656.09 & -0.005 & $\left({ }^{3} P\right)^{2} P_{3 / 2}-\left({ }^{3} F\right)^{2} D_{3 / 2}^{0}$ & 2058.946 & 100 & 48553.00 & 0.005 & $\left({ }^{1} D\right)^{2} D_{5 / 2}-(D)^{2} F_{5 / 2}^{0}$ \\
\hline 2901.176 & 1 & 34458.68 & 0.002 & $\left({ }^{1} S\right)^{2} S_{1 / 2}-\left({ }^{3} P\right)^{2} P_{3 / 2}^{0}$ & 2057.495 & 400 & 48587.24 & 0.001 & $\left({ }^{3} P\right)^{4} P_{5 / 2}-\left({ }^{3} P\right)^{4} S_{3 / 2}^{0}$ \\
\hline 2871.938 & 10 & 34809.47 & -0.003 & $\left({ }^{3} P\right)^{2} P_{1 / 2}-\left({ }^{3} F\right)^{2} D_{3 / 2}^{0}$ & 2055.999 & 2 & 48622.59 & -0.001 & $\left({ }^{1} G\right)^{2} G_{9 / 2}-\left({ }^{1} G\right)^{2} G_{7 / 2}^{0}$ \\
\hline 2823.509 & 5 & 35406.50 & -0.002 & $\left({ }^{1} D\right)^{2} D_{3 / 2}-\left({ }^{3} F\right)^{4} G_{5 / 2}^{0}$ & 2055.641 & 500 & 48.631 .05 & -0.001 & $\left({ }^{3} F\right)^{4} F_{7 / 2}-\left({ }^{3} F\right)^{4} G_{7 / 2}^{0}$ \\
\hline 2813.570 & 20 & 35531.56 & 0.004 & $\left({ }^{3} P\right)^{2} P_{3 / 2}-\left({ }^{3} F\right)^{4} D_{5 / 2}^{0}$ & 2054.391 & 200 & 48660.64 & 0.000 & $\left({ }^{3} P\right)^{4} P_{3 / 2}-\left({ }^{3} P\right)^{4} S_{3 / 2}^{0 / 2}$ \\
\hline 2749.577 & 1 & 36358.48 & -0.002 & $\left({ }^{1} D\right)^{2} D_{5 / 2}-\left({ }^{3} F\right)^{4} G_{7 / 2}^{0}$ & 2048.504 & 2 & 48800.46 & -0.017 & $\left({ }^{1} D\right)^{2} D_{3 / 2}^{3 / 2}-\left({ }^{l} D\right)^{2} P_{3 / 2}^{0}$ \\
\hline 2735.033 & 50 & 36551.81 & 0.005 & $\left({ }^{3} P\right)^{2} P_{3 / 2}-\left({ }^{3} F\right)^{2} D_{3 / 2}^{0}$ & 2044.252 & 100 & 48901.95 & 0.006 & $\left({ }^{3} F\right)^{2} F_{5 / 2}-\left({ }^{3} F\right)^{4} D_{7 / 2}^{0}$ \\
\hline 2727.272 & 100 & 36655.82 & 0.006 & $\left({ }^{\prime} G\right)^{2} G_{9 / 2}-\left({ }^{3} F\right)^{2} F_{7 / 2}^{0}$ & 2037.130 & 100 & 49072.89 & 0.008 & $\left({ }^{3} P\right)^{4} P_{1 / 2}-\left({ }^{3} P\right)^{4} S_{3 / 2}^{0}$ \\
\hline 2724.939 & 10 & 36687.20 & 0.000 & $\left({ }^{3} P\right)^{2} P_{1 / 2}-\left({ }^{3} F\right)^{4} D_{3,2}^{0}$ & 2028.300 & 600 & 49286.50 & 0.009 & $\left({ }^{3} F\right)^{2} F_{7 / 2}-\left({ }^{3} F\right)^{2} G_{9 / 2}^{0}$ \\
\hline 2712.486 & 5 & 36855.62 & 0.002 & $\left({ }^{1} G\right)^{2} G_{9 / 2}-\left({ }^{3} F\right)^{4} F_{9 / 2}^{0}$ & 2023.779 & 600 & 49396.59 & -0.001 & $\left({ }^{3} F\right)^{4} F_{9 / 2}-\left({ }^{3} F\right)^{4} G_{9 / 2}^{0}$ \\
\hline 2711.400 & 70 & 36870.38 & 0.003 & $\left({ }^{3} P\right)^{2} P_{3 / 2}-\left({ }^{3} P\right)^{2} S_{1 / 2}^{0}$ & 2022.657 & 5 & 49423.98 & 0.003 & $\left({ }^{1} D\right)^{2} D_{3 / 2}^{3 / 2}-\left({ }^{3} P\right)^{4} D_{3 / 2}^{0}$ \\
\hline 2648.126 & 1 & 37751.31 & -0.004 & $\left({ }^{1} D\right)^{2} D_{3 / 2}-\left({ }^{3} F\right)^{4} F_{3 / 2}^{0}$ & 2018.515 & 400 & 49525.39 & 0.001 & $\left({ }^{1} D\right)^{2} D_{3 / 2}-\left({ }^{1} D\right)^{2} F_{5 / 2}^{0}$ \\
\hline 2634.665 & 2 & 37944.17 & -0.001 & $\left({ }^{1} D\right)^{2} D_{5 / 2}-\left({ }^{3} F\right)^{4} F_{S / 2}^{0}$ & 2011.252 & 2 & 49704.21 & 0.001 & $\left({ }^{1} G\right)^{2} G_{7 / 2}-\left({ }^{3} P\right)^{4} P_{5 / 2}^{0}$ \\
\hline 2629.140 & 50 & 38.023 .91 & -0.006 & $\left({ }^{3} P\right)^{2} P_{1 / 2}-\left({ }^{3} P\right)^{2} S_{1 / 2}^{0}$ & 2010.889 & 700 & 49713.18 & 0.006 & $\left({ }^{3} F\right)^{4} F_{5 / 2}-\left({ }^{3} F\right)^{4} G_{7 / 2}^{0}$ \\
\hline 2568.836 & 10 & 38916.47 & -0.001 & $\left({ }^{1} D\right)^{2} D_{3 / 2}-\left({ }^{3} F\right)^{4} F_{5 / 2}^{0}$ & 2007.036 & 200 & 49808.60 & 0.003 & $\left({ }^{1} D\right)^{2} D_{5 / 2}-\left({ }^{3} P\right)^{4} D_{5 / 2}^{0}$ \\
\hline 2563.067 & 1 & 39004.06 & -0.010 & $\left({ }^{1} D\right)^{2} D_{5 / 2}-\left({ }^{3} F\right)^{2} F_{5 / 2}^{0}$ & 2006.411 & 500 & 49824.11 & 0.006 & $\left({ }^{3} F\right)^{2} F_{5 / 2}-\left({ }^{3} F\right)^{2} G_{7 / 2}^{0}$ \\
\hline 2556.567 & 10 & 39103.22 & 0.007 & $\left({ }^{3} P\right)^{4} P_{5 ; 2}-\left({ }^{3} F\right)^{4} G_{7 / 2}^{0}$ & 2000.700 & 200 & 49966.31 & 0.006 & $\left({ }^{1} D\right)^{2} D_{5 / 2}-\left({ }^{1} D\right)^{2} F_{7 / 2}^{0}$ \\
\hline 2511.251 & 20 & 39808.80 & -0.002 & $\left({ }^{1} G\right)^{2} G_{7 / 2}-\left({ }^{3} F\right)^{2} G_{7 / 2}^{0}$ & 1994.663 & 400 & 50133.78 & 0.002 & $\left({ }^{3} F\right)^{4} F_{5 / 2}-\left({ }^{3} F\right)^{4} F_{3 / 2}^{0}$ \\
\hline 2500.741 & 20 & 39976.09 & 0.007 & $\left({ }^{1} D\right)^{2} D_{3 / 2}-\left({ }^{3} F\right)^{2} F_{5 / 2}^{0}$ & 1991.374 & 400 & 50216.58 & 0.006 & $\left({ }^{3} F\right)^{4} F_{7 / 2}-\left({ }^{3} F\right)^{4} F_{5 / 2}^{0}$ \\
\hline 2484.509 & 100 & 40237.25 & -0.004 & $\left({ }^{3} F\right)^{2} F_{5 / 2}-\left({ }^{3} F\right)^{4} G_{5 / 2}^{0}$ & 1989.794 & 100 & 50256.45 & -0.001 & $\left({ }^{3} P\right)^{4} P_{3 / 2}-\left({ }^{3} P\right)^{4} D_{1 / 2}^{0}$ \\
\hline 2456.300 & 1 & 40699.31 & 0.009 & $\left({ }^{1} D\right)^{2} D_{3 / 2}-\left({ }^{3} F\right)^{2} D_{3 / 2}^{0}$ & 1988.645 & 200 & 50285.50 & -0.003 & $\left({ }^{1} S\right)^{2} S_{1 / 2}-\left({ }^{1} S\right)^{2} P_{1 / 2}^{0}$ \\
\hline 2451,374 & 50 & 40781.09 & -0.001 & $\left({ }^{1} D\right)^{2} D_{5 / 2}-\left({ }^{3} F\right)^{2} F_{7 / 2}^{0}$ & 1977.144 & 300 & 50578.00 & $0.00 \mathrm{l}$ & $\left({ }^{3} F\right)^{4} F_{9 / 2}-\left({ }^{3} F\right)^{4} F_{7 / 2}^{0}$ \\
\hline 2406.659 & 200 & 41538.74 & 0.001 & $\left({ }^{1} G\right)^{2} G_{9 / 2}-\left({ }^{3} F\right)^{2} G_{9 / 2}^{0}$ & 1974.494 & 300 & 50645.88 & 0.005 & $\left({ }^{3} P\right)^{4} P_{3 / 2}-\left({ }^{1} D\right)^{2} P_{3 / 2}^{0}$ \\
\hline 2405.019 & 20 & 41567.06 & -0.008 & $\left({ }^{3} F\right)^{2} F_{7 / 2}-\left({ }^{3} F\right)^{4} F_{5 / 2}^{0}$ & 1973.559 & 300 & 50668.85 & 0.002 & $\left({ }^{3} P\right)^{4} P_{1 / 2}-\left({ }^{3} P\right)^{4} D_{1 / 2}^{0}$ \\
\hline 2375.436 & 20 & 42084.68 & 0.002 & $\left({ }^{3} F\right)^{2} F_{7 / 2}-\left({ }^{3} F\right)^{4} G_{9 / 2}^{0}$ & 1971.114 & 200 & 50732.73 & 0.005 & $\left({ }^{l} D\right)^{2} D_{3 / 2}-\left({ }^{1} D\right)^{2} P_{L / 2}^{0}$ \\
\hline 2371.103 & 20 & 42161.58 & -0.007 & $\left({ }^{3} F\right)^{2} F_{5 / 2}-\left({ }^{3} F\right)^{4} G_{7 / 2}^{0}$ & 1971.045 & 600 & 50734.50 & 0.000 & $\left({ }^{3} F\right)^{4} F_{7 / 2}-\left({ }^{3} F\right)^{4} G_{9 / 2}^{0}$ \\
\hline 2353.752 & 20 & 42472.36 & -0.018 & $\left({ }^{3} P\right)^{4} P_{5 / 2}-\left({ }^{3} F\right)^{2} D_{3 / 2}^{0}$ & 1969.242 & 300 & 50780.96 & 0.001 & $\left({ }^{1} D\right)^{2} D_{3 / 2}-\left({ }^{3} P\right)^{4} D_{5 / 2}^{0}$ \\
\hline 2347.701 & 10 & 42581.82 & 0.007 & $\left({ }^{3} F\right)^{2} F_{5 / 2}-\left({ }^{3} F\right)^{4} F_{3 / 2}^{0}$ & 1966.043 & 400 & 50863.58 & 0.006 & $\left({ }^{3} F\right)^{4} F_{3 / 2}-\left({ }^{3} F\right)^{4} F_{3 / 2}^{0}$ \\
\hline 2310.560 & 1 & 43266.24 & -0.003 & $\left({ }^{3} F\right)^{2} F_{7 / 2}-\left({ }^{3} F\right)^{4} F_{7 / 2}^{0}$ & 1962.487 & 300 & 50955.75 & 0.001 & $\left({ }^{1} G\right)^{2} G_{7 / 2}-\left({ }^{1} G\right)^{2} H_{9 / 2}^{0}$ \\
\hline 2285.161 & 100 & 43747.09 & 0.004 & $\left({ }^{3} F\right)^{2} F_{5,2}-\left({ }^{3} F\right)^{4} F_{5,2}^{0}$ & 1959.130 & 200 & 51043.06 & 0.000 & $\left({ }^{1} G\right)^{2} G_{9 / 2}-\left({ }^{l} G\right)^{2} H_{9 / 2}^{0}$ \\
\hline 2276.496 & 1 & 43913.59 & 0.013 & $\left({ }^{1} D\right)^{2} D_{3 / 2}-\left({ }^{3} P\right)^{2} S_{\mathrm{l} / 2}^{0}$ & 1954.394 & 300 & 51166.75 & 0.004 & $\left({ }^{3} P\right)^{2} P_{3 / 2}-\left({ }^{3} P\right)^{2} D_{5 / 2}^{0}$ \\
\hline 2270.015 & 1 & 44038.95 & 0.003 & $\left({ }^{3} P\right)^{4} P_{1 / 2}-\left({ }^{3} F\right)^{4} D_{1 / 2}^{0}$ & 1953.255 & 20 & 51196.59 & 0.001 & $\left({ }^{3} P\right)^{4} P_{5 / 2}-\left({ }^{3} P\right)^{4} D_{3 / 2}^{0}$ \\
\hline 2251.363 & 300 & 44403.77 & 0.004 & $\left({ }^{3} F\right)^{2} F_{7 / 2}-\left({ }^{3} F\right)^{2} F_{7 / 2}^{0}$ & 1950.760 & 200 & 51262.07 & 0.002 & $\left({ }^{1} D\right)^{2} D_{5 ; 2}-\left({ }^{1} D\right)^{2} D_{3 / 2}^{0}$ \\
\hline 2250.495 & 2 & 44420.90 & 0.005 & $\left({ }^{3} P\right)^{4} P_{3 / 2}-\left({ }^{3} F\right)^{4} D_{5 / 2}^{0}$ & 1950.460 & 300 & 51269.95 & 0.002 & $\left({ }^{3} P\right)^{4} P_{3 / 2}^{5 / 2}-\left({ }^{3} P\right)^{4} D_{3 / 2}^{0}$ \\
\hline 2250.391 & 1 & 44422.95 & 0.011 & $\left({ }^{3} P\right)^{4} P_{3 / 2}-\left({ }^{3} F\right)^{4} D_{3 / 2}^{0}$ & 1950.214 & 50 & 51276.42 & 0.002 & $\left({ }^{3} F\right)^{4} F_{7 / 2}-\left({ }^{3} F\right)^{2} F_{3 / 2}^{0}$ \\
\hline 2241.281 & 5 & 44603.49 & 0.005 & $\left({ }^{3} F\right)^{2} F_{7 / 2}-\left({ }^{3} F\right)^{4} F_{9 / 2}^{0}$ & 1949.360 & 500 & 51298.88 & 0.006 & $\left({ }^{3} F\right)^{4} F_{S / 2}-\left({ }^{3} F\right)^{4} F_{S / 2}^{0}$ \\
\hline 2231.106 & 500 & 44806.89 & 0.001 & $\left({ }^{3} F\right)^{2} F_{5 / 2}-\left({ }^{3} F\right)^{2} F_{5 / 2}^{0}$ & 1946.610 & 200 & 51371.35 & 0.000 & $\left({ }^{3} P\right)^{4} P_{3 / 2}^{5 / 2}-\left({ }^{1} D\right)^{2} F_{5 / 2}^{0}$ \\
\hline 2229.676 & 1 & 44835.62 & 0.000 & $\left({ }^{3} P\right)^{4} P_{1 / 2}-\left({ }^{3} F\right)^{4} D_{3 / 2}^{0}$ & 1944.197 & 300 & 51435.11 & 0.005 & $\left({ }^{\prime} D\right)^{2} D_{5 / 2}-\left({ }^{3} P\right)^{4} D_{7 / 2}^{0}$ \\
\hline 2210.463 & 50 & 45225.29 & 0.011 & $\left({ }^{3} F\right)^{2} F_{7 / 2}-\left({ }^{3} F\right)^{4} D_{5 / 2}^{0}$ & 1936.283 & 200 & 51645.34 & 0.002 & $\left({ }^{3} F\right)^{2} F_{5 / 2}-\left({ }^{3} P\right)^{4} S_{3 / 2}^{0}$ \\
\hline 2203.525 & 20 & 45367.67 & 0.010 & $\left({ }^{3} P\right)^{4} P_{5 / 2}-\left({ }^{3} F\right)^{2} D_{5 / 2}^{0}$ & 1934.899 & 400 & 51682.28 & 0.007 & $\left({ }^{3} P\right)^{4} P_{1 / 2}-\left({ }^{3} P\right)^{4} D_{3 / 2}^{0}$ \\
\hline 2199.958 & 2 & 45441.22 & 0.001 & $\left({ }^{3} P\right)^{4} P_{3 / 2}-\left({ }^{3} F\right)^{2} D_{5 / 2}^{0}$ & 1933.653 & 400 & 51715.58 & 0.004 & $\left({ }^{3} F\right)^{4} F_{9 / 2}-\left({ }^{3} F\right)^{2} F_{7 / 2}^{0}$ \\
\hline 2195.678 & 100 & 45529.79 & 0.018 & $\left({ }^{3} F\right)^{2} F_{5 / 2}-\left({ }^{3} F\right)^{2} D_{3 / 2}^{0}$ & 1932.906 & 300 & 51735.57 & 0.000 & $\left({ }^{1} D\right)^{2} D_{5 / 2}-\left({ }^{1} D\right)^{2} D_{5 / 2}^{0}$ \\
\hline 2187.110 & 1 & 45708.13 & 0.006 & $\left({ }^{3} P\right)^{2} P_{3: 2}-\left({ }^{3} P\right)^{4} P_{1 / 2}^{0}$ & 1930.467 & 300 & 51800.93 & 0.003 & $\left({ }^{3} P\right)^{2} D_{3 / 2}^{0}$ \\
\hline 2184.917 & 20 & 45754.01 & -0.002 & $\left({ }^{1} G\right)^{2} G_{7 / 2}-\left({ }^{1} D\right)^{2} F_{7 / 2}^{0}$ & 1929.210 & 900 & 51834.68 & -0.001 & $\left({ }^{3} F\right)^{4} F_{9 / 2}-\left({ }^{3} F\right)^{4} G_{11 / 2}^{0}$ \\
\hline 2180.634 & 100 & 45843.86 & 0.005 & $\left({ }^{3} P\right)^{4} P_{5 / 2}-\left({ }^{3} F\right)^{4} D_{7 / 2}^{0}$ & 1926.200 & 800 & 51915.68 & -0.009 & $\left.\left({ }^{3} F\right)^{4} F_{9 / 2}-\left({ }^{3} F\right)^{4} F_{9 / 2}^{0}\right\}$ \\
\hline 2167.144 & 200 & 46129.20 & -0.010 & $\left({ }^{3} P\right)^{2} P_{3 / 2}-\left({ }^{3} P\right)^{4} P_{3 / 2}^{0}$ & & & & 0.011 & $\left.\left({ }^{3} F\right)^{4} F_{7 / 2}-\left({ }^{3} F\right)^{4} F_{7 / 2}^{0}\right\}$ \\
\hline 2161.680 & 200 & 46245.79 & -0.001 & $\left({ }^{3} F\right)^{2} F_{7 / 2}-\left({ }^{3} F\right)^{2} D_{5 / 2}^{0}$ & 1922.008 & 200 & 52028.92 & 0.001 & $\left({ }^{3} F\right)^{4} F_{3 / 2}-\left({ }^{3} F\right)^{4} F_{5 / 2}^{0}$ \\
\hline 2157.099 & 20 & 46343.99 & 0.019 & $\left({ }^{3} P\right)^{2} P_{1 / 2}-\left({ }^{1} D\right)^{2} D_{3 / 2}^{0}$ & 1916.597 & 10 & 52175.81 & 0.003 & $\left({ }^{3} F\right)^{2} F_{7 / 2}-\left({ }^{1} D\right)^{2} F_{5 / 2}^{0}$ \\
\hline 2145.976 & 2 & 46584.17 & -0.004 & $\left({ }^{3} F\right)^{2} F_{5 / 2}-\left({ }^{3} F\right)^{2} F_{7,2}^{0}$ & 1915.709 & 200 & 52199.99 & 0.005 & $\left({ }^{1} D\right)^{2} D_{5 / 2}-\left({ }^{3} P\right)^{4} P_{3 / 2}^{0}$ \\
\hline 2139.651 & 100 & 46721.86 & 0.000 & $\left({ }^{3} F\right)^{2} F_{7 / 2}-\left({ }^{3} F\right)^{4} D_{7 / 2}^{0}$ & 1914.441 & 500 & 52234.56 & -0.005 & $\left({ }^{1} D\right)^{2} D_{3 / 2}-\left({ }^{1} D\right)^{2} D_{3 / 2}^{0}$ \\
\hline 2137.614 & 1 & 46766.38 & -0.012 & $\left({ }^{3} P\right)^{4} P_{5 / 2}-\left({ }^{3} F\right)^{2} G_{7 / 2}^{0}$ & 1911.690 & 300 & 52309.73 & 0.002 & $\left({ }^{3} P\right)^{2} P_{3 / 2}-\left({ }^{3} P\right)^{2} P_{1 / 2}^{0}$ \\
\hline 2135.399 & 1 & 46814.88 & -0.009 & $\left({ }^{1} D\right)^{2} D_{3 ; 2}-\left({ }^{3} P\right)^{4} S_{3 / 2}^{0}$ & 1909.904 & 400 & 52358.65 & 0.005 & $\left({ }^{3} F\right)^{4} F_{5 / 2}-\left({ }^{3} F\right)^{2} F_{5 / 2}^{0}$ \\
\hline 2116.955 & 100 & 47222.71 & 0.002 & $\left({ }^{1} G\right)^{2} G_{7 / 2}-\left({ }^{3} P\right)^{4} D_{7 / 2}^{0}$ & 1902.821 & 300 & 52553.55 & 0.000 & $\left({ }^{3} P\right)^{4} P_{5 / 2}-\left({ }^{3} P\right)^{4} D_{5 / 2}^{0}$ \\
\hline 2113.040 & 5 & 47310.19 & -0.006 & $\left({ }^{\prime} G\right)^{2} G_{9 / 2}-\left({ }^{3} P\right)^{4} D_{7 / 2}^{0}$ & 1901.905 & 200 & 52578.86 & -0.002 & $-\left({ }^{1} D\right)^{2} P_{1 / 2}^{0}$ \\
\hline 2108.775 & 20 & 47405.87 & -0.006 & $\left({ }^{3} F\right)^{2} F_{5,2}-\left({ }^{3} F\right)^{4} F_{5,2}^{0}$ & 1900.165 & 400 & 52627.00 & -0.003 & $\left({ }^{3} P\right)^{4} P_{3 / 2}-\left({ }^{3} P\right)^{4} D_{5 / 2}^{0}$ \\
\hline 2098.250 & 200 & 47643.63 & 0.017 & $\left({ }^{3} F\right)^{2} F_{7 / 2}-\left({ }^{3} F\right)^{2} G_{7 / 2}^{0}$ & 1897.249 & 200 & 52707.89 & -0.001 & $\left({ }^{1} D\right)^{2} D_{3 / 2}-\left({ }^{1} D\right)^{2} D_{5 / 2}^{0}$ \\
\hline 2091.864 & 400 & 47789.06 & 0.000 & $\left({ }^{3} F\right)^{4} F_{5 / 2}-\left({ }^{3} F\right)^{4} G_{5 / 2}^{0}$ & 1897.128 & 400 & 52711.25 & 0.003 & $\left({ }^{3} P\right)^{4} P_{5 / 2}-\left({ }^{1} D\right)^{2} F_{7 / 2}^{0}$ \\
\hline 2091.142 & 200 & 47.805 .56 & -0.001 & $\left({ }^{\mathrm{l}} G\right)^{2} G_{7 / 2}-\left({ }^{\mathrm{l}} G\right)^{2} G_{9 / 2}^{0}$ & 1895.813 & 400 & 52747.81 & -0.003 & $\left({ }^{i} D\right)^{2} D_{5 ; 2}-\left({ }^{1} G\right)^{2} G_{7 / 2}^{0}$ \\
\hline 2090.169 & 300 & 47827.81 & -0.002 & $\left({ }^{1} D\right)^{2} D_{5 / 2}-\left({ }^{1} D\right)^{2} P_{3 / 2}^{0}$ & 1895.675 & 200 & 52751.65 & 0.001 & $\left({ }^{1} D\right)^{2} D_{3 / 2}-\left({ }^{3} P\right)^{4} P_{1 / 2}^{0}$ \\
\hline 2089.390 & 2 & 47845.64 & -0.003 & $\left({ }^{3} P\right)^{2} P_{3 / 2}-\left({ }^{3} P\right)^{4} P_{5 / 2}^{0}$ & 1890.140 & 800 & 52906.13 & -0.001 & $\left({ }^{1} G\right)^{2} G_{9 / 2}-\left({ }^{1} G\right)^{2} H_{11 / 2}^{0}$ \\
\hline 2087.328 & 500 & 47892.90 & -0.003 & $\left({ }^{1} G\right)^{2} G_{9 / 2}-\left({ }^{1} G\right)^{2} G_{9 / 2}^{0}$ & 1887.734 & 300 & 52973.57 & -0.002 & $\left({ }^{\prime} S\right)^{2} S_{1 / 2}-\left({ }^{1} S\right)^{2} P_{3 / 2}^{0}$ \\
\hline 2065.010 & 20 & 48410.44 & 0.002 & $\left({ }^{1} D\right)^{2} D_{3,2}-\left({ }^{3} P\right)^{4} D_{1 / 2}^{0}$ & 1887.101 & 20 & 52991.33 & -0.003 & $\left({ }^{3} P\right)^{4} P_{1 / 2}-\left({ }^{1} D\right)^{2} P_{1 / 2}^{0}$ \\
\hline 2064.345 & 10 & 48426.03 & -0.001 & $\left({ }^{3} F\right)^{2} F_{5 / 2}-\left({ }^{3} F\right)^{2} D_{3,2}^{0}$ & 1886.847 & 500 & 52998.46 & -0.006 & $\left({ }^{3} F\right)^{4} F_{5 / 2}-\left({ }^{3} F\right)^{4} F_{7 / 2}^{0}$ \\
\hline 2063.249 & 300 & 48451.75 & 0.000 & $\left({ }^{1} D\right)^{2} D_{5 ; 2}-\left({ }^{3} P\right)^{4} D_{3 / 2}^{0}$ & 1885.885 & 10 & 53025.50 & 0.000 & $\left({ }^{1} G\right)^{2} G_{7 / 2}-\left({ }^{3} P\right)^{2} D_{5 / 2}^{0}$ \\
\hline
\end{tabular}

Table I. Continued 
Table I. Continued

\begin{tabular}{|c|c|c|c|c|c|c|c|c|c|}
\hline $\begin{array}{l}\text { Wavelength } \\
(\AA)\end{array}$ & Int. & $\begin{array}{l}\text { Wavenumber } \\
\left(\mathrm{cm}^{-1}\right)\end{array}$ & $O-C(\AA)$ & $\begin{array}{l}\text { Classification } \\
4 d^{3}, 4 d^{2}() 5 s-4 d^{2}() 5 p\end{array}$ & $\begin{array}{l}\text { Wavelength } \\
(\AA)\end{array}$ & Int. & $\begin{array}{l}\text { Wavenumber } \\
\left(\mathrm{cm}^{-1}\right)\end{array}$ & $O-C(\AA)$ & $\begin{array}{l}\text { Classification } \\
4 d^{3}, 4 d^{2}() 5 s-4 d^{2}() 5 p\end{array}$ \\
\hline 1884.883 & 300 & 53053.69 & -0.001 & $\left({ }^{3} F\right)^{4} F_{7 / 2}-\left({ }^{3} F\right)^{2} F_{7: 2}^{0}$ & 1674.491 & 5 & 59719.64 & 0.002 & $\left({ }^{3} F\right)^{2} F_{50}-\left({ }^{3} P\right)^{4} P_{5}^{0}$ \\
\hline 1883.875 & 200 & 53082.07 & -0.001 & $\left({ }^{3} F\right)^{4} F_{5 / 2}-\left({ }^{3} F\right)^{2} D_{3 ! 2}^{0}$ & 1668.072 & 10 & 59949.45 & 0.000 & $\left({ }^{3} P\right)^{4} P_{1 / 2}-\left({ }^{3} P\right)^{2} D_{3,2}^{0}$ \\
\hline 1883.642 & 500 & 53088.64 & 0.002 & $\left({ }^{3} F\right)^{4} F_{3 / 2}-\left({ }^{3} F\right)_{5 / 2}^{0}$ & & & & & \\
\hline 1880.685 & 300 & 53172.11 & 0.011 & $\left({ }^{1} D\right)^{2} D_{3 / 2}-\left({ }^{3} P\right)^{4} P_{3: 2}^{0}$ & 1660.198 & 20 & 60233.77 & -0.001 & $\left({ }^{(} D\right)^{-} D_{2 / 3}-\left({ }^{(} P\right)^{-} P_{3 / 2}^{0}$ \\
\hline 1880.043 & 500 & 53190.27 & 0.001 & $\left({ }^{3} P\right)^{2} P_{3 / 2}-\left({ }^{3} P\right)^{2} P_{3 / 2}^{0}$ & 1644.038 & 200 & 60825.84 & -0.004 & $\left({ }^{3} F\right)^{4} F_{7 / 2}-\left({ }^{1} D\right)^{2} F_{5 / 2}^{0}$ \\
\hline 1877.814 & 700 & 53253.41 & 0.000 & $\left({ }^{3} F\right)^{4} F_{32}-\left({ }^{3} F\right)^{4} F_{9}^{0}$ & 1643.090 & 300 & 60860.93 & -0.005 & $\left({ }^{3} F\right)^{2} F_{7 / 2}-\left({ }^{3} P\right)^{2} D_{5 ; 2}^{0}$ \\
\hline 1871.557 & 100 & 53431.44 & 0.000 & $\left({ }^{3} F\right)^{2} F_{70}-\left({ }^{3} P\right)^{4} D_{5}^{0}$ & 1642.005 & 200 & 60901.15 & -0.002 & $\left({ }^{3} F\right)^{4} F_{9 / 2}-\left({ }^{1} D\right)^{2} F_{7 / 2}^{0}$ \\
\hline 1870.446 & 300 & 53463.18 & 0.000 & $\left({ }^{3} P\right)^{2} P^{7 / 2}-\left({ }^{3} P\right)^{2} P^{0}$ & 1634.460 & 200 & 61182.28 & 0.010 & $\left({ }^{3} F\right)^{4} F_{5 / 2}-(D)^{2} P_{3 / 2}^{0}$ \\
\hline & & & 0.000 & & 1634.008 & 10 & 61199.21 & -0.001 & $\left({ }^{3} P\right)^{4} P_{3 / 2}-\left({ }^{3} P\right)^{2} P_{1 ; 2}^{0}$ \\
\hline 1866.047 & 400 & 53589.21 & 0.000 & $\left({ }^{3} F\right)^{2} F_{7,2}-\left({ }^{1} D\right)^{2} F_{7 / 2}^{0}$ & 1625.406 & 300 & 61523.09 & -0.003 & $\left({ }^{3} F\right)^{4} F_{3 / 2}^{3 / 2}-\left({ }^{3} P\right)^{4} D_{1 / 2}^{0}$ \\
\hline 1864.605 & 300 & 53630.66 & 0.004 & $\left({ }^{3} F\right)^{2} F_{5 / 2}-\left({ }^{1} D\right)^{2} P_{3 / 2}^{0}$ & 1623.067 & 20 & 61611.75 & -0.003 & $\left({ }^{3} P\right)^{4} P_{3 / 2}-\left({ }^{3} P\right)^{2} P_{1 / 2}^{0}$ \\
\hline 1858.328 & 600 & 53811.81 & 0.005 & $\left({ }^{3} F\right)^{4} F_{3 / 2}-\left({ }^{3} F\right)^{2} D_{3 / 2}^{0}$ & 1617.950 & 300 & 61806.60 & 0.001 & $\left({ }^{3} F\right)^{4} F_{5 / 2}-\left({ }^{3} P\right)^{4} D_{3,2}^{0}$ \\
\hline 1856.137 & 500 & 53875.33 & -0.001 & $\left({ }^{3} F\right)^{4} F_{3: 2}-\left({ }^{3} F\right)^{4} D_{5 / 2}^{0}$ & 1615.298 & 20 & 61908.08 & -0.002 & $\left({ }^{3} F\right)^{4} F_{3 / 2}-\left({ }^{1} D\right)^{2} F_{S / 2}^{(0}$ \\
\hline 1851.613 & 100 & 54006.96 & 0.001 & $\left({ }^{3} P\right)^{4} P_{S / 2}-(D)^{2} D_{3 / 2}^{0}$ & & & & & $\left({ }^{3} F\right)^{4} F_{3,0}-\left({ }^{1} D\right)^{2} P^{0}$ \\
\hline 1850.700 & 700 & 54033.60 & 0.003 & $\left({ }^{3} F\right)^{4} F_{9 ; 2}-\left({ }^{3} F\right)^{4} D_{7: 2}^{0}$ & $\begin{array}{l}1615.186 \\
1610788\end{array}$ & $\begin{array}{r}20 \\
200\end{array}$ & 62081.41 & $\begin{array}{r}0.005 \\
-0.004\end{array}$ & $\begin{array}{l}-(D)^{2} P_{3 / 2} \\
-\left({ }^{3} P\right)^{4} D_{5}^{0}\end{array}$ \\
\hline $1849.09 \mathrm{l}$ & 200 & 54080.62 & -0.009 & $\left({ }^{3} P\right)^{4} P_{3 ; 2}-\left({ }^{1} D\right)^{2} D_{3: 2}^{0}$ & 1610.788 & 200 & $\begin{array}{l}62081.41 \\
62038.94\end{array}$ & -0.004 & $\begin{array}{l}\left({ }^{(} F\right)^{4} F_{7 / 2}-(3 P)^{4} D_{5 / 2}^{0} \\
\left.{ }^{3} F\right)^{4} F\end{array}$ \\
\hline 1847.198 & 400 & 54136.04 & -0.003 & $\left({ }^{3} F\right)^{4} F_{5 / 2}-\left({ }^{3} F\right)^{2} F_{7 / 2}^{0}$ & 1606.711 & 50 & 62238.94 & 0.002 & $\left({ }^{3} F\right)^{4} F_{7 / 2}-\left({ }^{1} D\right)^{2} F_{7 / 2}^{0}$ \\
\hline 1845.690 & 700 & 54180.27 & -0.005 & $\left({ }^{3} P\right)^{4} P_{5 / 2}-\left({ }^{3} P\right)^{4} D_{7 / 2}^{0}$ & 1604.734 & 100 & 62315.62 & 0.001 & $\left({ }^{1} D\right)^{2} D_{3 / 2}-\left({ }^{1} G\right)^{2} F_{5 / 2}^{0}$ \\
\hline 1843.140 & 20 & 54255.23 & -0.016 & $\left({ }^{3} F\right)^{2} F_{3 / 2}-\left({ }^{3} P\right)^{4} D_{3 / 2}^{0}$ & 1603.342 & 200 & 62369.72 & 0.004 & $\left({ }^{3} F\right)^{4} F_{9 ; 2}-\left({ }^{3} P\right)^{4} D_{7 ; 2}^{0}$ \\
\hline & & & & & 1600.200 & 10 & 62492.18 & -0.001 & $\left({ }^{3} P\right)^{4} P_{1,2}-\left({ }^{3} P\right)^{2} P_{3,2}^{0}$ \\
\hline 1840.136 & 10 & 54343.80 & -0.003 & $\left({ }^{3} P\right)^{2} P_{1 / 2}-\left({ }^{3} P\right)^{2} P_{3 ; 2}^{0}$ & 1599.442 & 200 & 62521.80 & -0.001 & $\left({ }^{3} F\right)^{2} F_{s_{2}}-\left({ }^{3} P\right)^{2} D_{3 / 2}^{0}$ \\
\hline 1839.714 & 300 & $543 \leq 6.27$ & -0.005 & $\left({ }^{3} F\right)^{2} F_{5 / 2}-\left({ }^{1} D\right)^{2} F_{5 / 2}^{0}$ & 1599.069 & 100 & 62536.38 & 0.004 & $\left({ }^{3} F\right)^{4} F_{3 / 2}-\left({ }^{3} P\right)^{4} D_{3,2}^{0}$ \\
\hline 1835.521 & 400 & 54480.44 & 0.000 & $\left({ }^{3} P\right)^{4} P_{5 / 2}-(D)^{2} D_{5: 2}^{0}$ & 1594.521 & 20 & 62714.75 & 0.003 & $\left({ }^{3} P\right)^{4} P_{5 / 2}-\left({ }^{1} G\right)^{2} F_{7 / 2}^{0}$ \\
\hline 1835.094 & 200 & 54493.12 & -0.010 & $\left({ }^{3} P\right)^{4} P_{1 / 2}-\left({ }^{1} D\right)^{2} D_{3 / 2}^{0}$ & 1588.500 & 10 & 62952.47 & 0.004 & $\left({ }^{3} F\right)^{4} F_{9 / 2}-\left({ }^{1} G\right)^{2} G_{9 / 2}^{0}$ \\
\hline 1833.047 & 600 & 54553.97 & -0.005 & $\left({ }^{3} P\right)^{4} P_{3 / 2}-\left({ }^{1} D\right)^{2} D_{5 / 2}^{0}$ & & & & & \\
\hline 1831.575 & 500 & 54597.81 & -0.006 & $\left({ }^{3} P\right)^{4} P_{3 / 2}-\left({ }^{3} P\right)^{4} P_{1 / 2}^{0}$ & 1586.270 & 20 & 63040.97 & 0.001 & $\left({ }^{3} F\right)^{2} F_{5 / 2}-\left({ }^{3} P\right)^{2} D_{5 / 2}^{0}$ \\
\hline 1821.853 & 300 & 54889.17 & -0.005 & $\left({ }^{1} D\right)^{2} D_{3 / 2}-\left({ }^{3} P\right)^{4} P_{5 / 2}^{0}$ & 1583.192 & 200 & 63163.53 & 0.001 & $\left({ }^{3} F\right)^{4} F_{5: 2}-\left({ }^{3} P\right)^{4} D_{5 / 2}^{0}$ \\
\hline 1821.718 & 500 & 54893.23 & -0.005 & $\left({ }^{3} F\right)^{4} F_{32}-\left({ }^{3} F\right)^{4} D^{0}$ & 1572.511 & 5 & 63592.56 & 0.005 & $\left({ }^{3} F\right)^{2} F_{7 / 2}-\left({ }^{l} G\right)^{2} F_{7 / 2}^{0}$ \\
\hline 1821.635 & 500 & 54895.73 & -0.005 & $\left({ }^{3} F\right)^{4} F_{7,}-\left({ }^{3} F\right)^{2} D^{0}$ & 1570.300 & 20 & 63682.09 & 0.006 & $\left({ }^{3} F\right)^{4} F_{9 / 2}-\left({ }^{\prime} G\right)^{2} G_{7 / 2}^{0}$ \\
\hline 1820.005 & 300 & 54944.90 & 0.003 & $\left({ }^{3} P\right)^{4} P_{5,2}-\left({ }^{3} P\right)^{4} P_{3,2}^{0}$ & 1569.672 & 50 & 63707.57 & 0.006 & $\left({ }^{3} F\right)^{4} F_{7,2}-\left({ }^{3} P\right)^{4} D_{7,2}^{0}$ \\
\hline & & & & $(1) 1_{5 / 2}(1) l_{3 / 2}$ & 1566.281 & 5 & 63845.50 & -0.004 & $\left({ }^{3} F\right)^{4} F_{3 / 2}-\left({ }^{1} F\right)^{2} P_{\mathrm{t} / 2}^{0}$ \\
\hline 1819.575 & 500 & 54957.88 & -0.009 & $\left({ }^{3} F\right)^{4} F_{5 / 2}-\left({ }^{3} F\right)^{4} D_{5 ; 2}^{0}$ & 1565.110 & 10 & 63893.27 & 0.005 & $\left({ }^{3} F\right)^{4} F_{3 ; 2}-\left({ }^{3} P\right)^{4} D_{5 / 2}^{0}$ \\
\hline 1819.500 & 600 & 54960.15 & -0.011 & $\left({ }^{3} F\right)^{4} F_{5 / 2}-\left({ }^{3} F\right)^{4} D_{3 ; 2}^{0}$ & 1562.312 & 100 & 64007.70 & 0.011 & $\left({ }^{3} F\right)^{4} F_{7 ; 2}-\left({ }^{1} D\right)^{2} D_{5 ; 2}^{0}$ \\
\hline 1817.842 & 200 & 55010.28 & -0.006 & $\left({ }^{3} P\right)^{4} P_{1 ; 2}-\left({ }^{3} P\right)^{4} P_{1 ; 2}^{0}$ & 1555.451 & 50 & 64.290 .03 & 0.013 & $\left({ }^{3} F\right)^{4} F_{7 / 2}-\left({ }^{1} G\right)^{2} G_{9 / 2}^{0}$ \\
\hline 1817.572 & 200 & 55018.45 & -0.003 & $\left({ }^{3} P\right)^{4} P_{3 / 2}-\left({ }^{3} P\right)^{4} P_{3 / 2}^{0}$ & 1543.457 & 10 & 64789.62 & 0.012 & $\left({ }^{3} F\right)^{4} F_{5 ; 2}-\left({ }^{3} P\right)^{4} D D_{7 / 2}^{0}$ \\
\hline 1816.251 & 5 & 55058.46 & -0.015 & $\left({ }^{3} F\right)^{2} F_{7 / 2}-\left({ }^{3} P\right)^{4} D_{7 / 2}^{0}$ & & & & & \\
\hline 1809.219 & 50 & 55272.46 & -0.008 & $\left({ }^{3} P\right)^{2} P_{3: 2}-\left({ }^{1} G\right)^{2} F_{5 / 2}^{0}$ & 1536.944 & 100 & $\begin{array}{l}65004.17 \\
65090.05\end{array}$ & & $(F)^{2} F_{5 ; 2}-(P)^{4} P_{3 / 2}^{3}$ \\
\hline 1806.405 & 200 & 55358.57 & -0.008 & $\left({ }^{3} F\right)^{2} F_{7 / 2}-\left({ }^{1} D\right)^{2} D_{s / 2}^{0}$ & 1536.333 & 2 & $\begin{array}{l}65090.05 \\
65346.66\end{array}$ & 0.009 & $J^{4} F_{5 ; 2}-\left({ }^{1} D\right)^{2} D_{5 / 2}^{0}$ \\
\hline 1805.964 & 500 & 55372.08 & -0.014 & $\left({ }^{3} F\right)^{4} F_{7 / 2}-\left({ }^{3} F\right)^{4} D_{7 / 2}^{0}$ & 1530.300 & 5 & 65346.66 & 0.006 & $\left({ }^{3} F\right)^{4} F_{3 / 2}-\left({ }^{1} D\right)^{2} D_{3 / 2}^{0}$ \\
\hline 1804.047 & 500 & 55430.92 & -0.003 & $\left({ }^{3} P\right)^{4} P_{1,2}-\left({ }^{3} P\right)^{4} P_{3 / 2}^{0}$ & 1525.431 & 50 & 65555.24 & -0.005 & $\left({ }^{3} F\right)^{4} F_{5 / 2}-\left({ }^{3} P\right)^{4} P_{3 / 2}^{0}$ \\
\hline 1797.233 & 500 & 55641.08 & -0.010 & $\left({ }^{3} F\right)^{2} F_{7,2}-\left({ }^{l} G\right)^{2} G_{9,2}^{0}$ & 1520.391 & 20 & 65772.55 & 0.010 & $\left({ }^{3} F\right)^{2} F_{5: 2}-\left({ }^{1} G\right)^{2} F_{7 / 2}^{0}$ \\
\hline & & & & & 1489.292 & 20 & 67146.00 & 0.008 & $\left({ }^{3} F\right)^{2} F_{5: 2}-\left({ }^{1} G\right)^{2} F_{s: 2}^{0}$ \\
\hline 1795.726 & 50 & 55687.78 & -0.008 & $\left({ }^{3} F\right)^{4} F_{3 ; 2}-\left({ }^{3} F\right)^{4} D_{5 / 2}^{0}$ & 1425.103 & 100 & 70170.37 & 0.001 & $\left({ }^{3} P\right)^{2} P_{1 / 2}-\left({ }^{1} S\right)^{2} P_{1 / 2}^{0}$ \\
\hline 1795.651 & 200 & 55690.10 & -0.013 & $\left({ }^{3} F\right)^{4} F_{3 ; 2}-\left({ }^{3} F\right)^{4} D_{3 ; 2}^{0}$ & 1424.811 & 50 & 70184.74 & 0.000 & ${ }^{2} D 1_{3 / 2}-\left({ }^{3} F\right)^{4} G_{5 / 2}^{0}$ \\
\hline 1793.476 & 300 & 55757.64 & -0.005 & $\left({ }^{1} G\right)^{2} G_{7 / 2}-\left({ }^{1} G\right)^{2} F_{7: 2}^{0}$ & 1412.367 & 300 & 70803.12 & 0.008 & $\left({ }^{3} F\right)^{4} F_{3 / 2}-\left({ }^{3} P\right)^{2} D_{3 / 2}^{0}$ \\
\hline 1793.089 & 300 & 55769.68 & -0.007 & $\left({ }^{3} F\right)^{2} F_{5,2}-\left({ }^{1} D\right)^{2} F_{7 / 2}^{0}$ & 1410.339 & 50 & 70904.93 & -0.007 & $\left({ }^{3} F\right)^{4} F_{9,2}-\left({ }^{1} G\right)^{2} F_{7: 2}^{0}$ \\
\hline 1790.672 & 700 & 55844.95 & -0.005 & $\left({ }^{3} G\right)^{2} G_{9: 2}-\left({ }^{\prime} G\right)^{2} F_{7,2}^{0}$ & & & & & \\
\hline 1786.418 & 300 & 55977.94 & -0.002 & $\left({ }^{3} F\right)^{4} F_{5: 2}-\left({ }^{3} F\right)^{2} D_{5: 2}^{0}$ & 1394.604 & 2 & 71704.94 & 0.002 & $\left({ }^{(} P\right)^{2} P_{3 ; 2}-\left({ }^{1} S\right)^{2} P_{3 ; 2}^{0}$ \\
\hline 1776.385 & 300 & 56294.10 & -0.009 & $\left({ }^{3} F\right)^{4} F_{7 / 2}-\left({ }^{3} F\right)^{2} G_{7 / 2}^{0}$ & 1378.751 & 5 & 72529.41 & 0.002 & ${ }^{2} D \mathrm{I}_{3 / 2}-\left({ }^{3} F\right)^{4} F_{3 / 2}^{0}$ \\
\hline 1773.974 & 200 & 56370.61 & -0.003 & $\left({ }^{3} F\right)^{2} F_{7 / 2}-\left({ }^{1} G\right)^{2} G_{7 / 2}^{0}$ & 1363.791 & 10 & 73325.01 & -0.003 & $\left({ }^{3} F\right)^{4} F_{5 ; 2}-\left({ }^{1} G\right)^{2} F_{7: 2}^{0}$ \\
\hline 1766.826 & 300 & 56598.66 & -0.003 & $\left({ }^{3} F\right)^{4} F_{9 ; 2}-\left({ }^{3} F\right)^{2} G_{9 / 2}^{0}$ & 1337.710 & 50 & 74754.61 & -0.003 & ${ }^{2} D 1_{3 ; 2}-\left({ }^{3} F\right)^{2} F_{5 / 2}^{0}$ \\
\hline 1764.866 & 200 & 56661.52 & 0.002 & $\left({ }^{3} P\right)^{4} P_{5,2}-\left({ }^{3} P\right)^{4} P_{5 ; 2}^{0}$ & 1324.892 & 50 & 75477.85 & -0.002 & ${ }^{2} D 1_{3 / 2}-\left({ }^{3} F\right)^{2} D_{3 / 2}^{0}$ \\
\hline & & & & & 1301.402 & 10 & 76840.20 & -0.006 & ${ }^{2} D 1_{5 ; 2}\left({ }^{3} F\right)^{2} F_{7 / 2}^{0}$ \\
\hline 1763.423 & 50 & 56707.89 & -0.003 & $\left({ }^{3} F\right)^{4} F_{3 / 2}-\left({ }^{3} F\right)^{2} D_{5 / 2}^{0}$ & 1292.743 & 20 & 77354.89 & 0.010 & ${ }^{2} D 1_{3 / 2}-\left({ }^{3} F\right)^{4} D_{3 / 2}^{0}$ \\
\hline 1763.087 & 50 & 56718.69 & 0.002 & $\left({ }^{1} D\right)^{2} D_{5 / 2}-\left({ }^{3} P\right)^{2} D_{3 / 2}^{0}$ & 1287.641 & 5 & 77661.39 & 0.002 & ${ }^{2} D 1_{5 / 2}-\left({ }^{3} F\right)^{4} D_{5,2}^{0}$ \\
\hline 1762.581 & 300 & 56734.98 & -0.001 & $\left({ }^{3} P\right)^{4} P_{3 / 2}-\left({ }^{3} P\right)^{4} P_{5 / 2}^{0}$ & 1285.748 & 5 & 77775.73 & 0.007 & $\left({ }^{1} D\right)^{2} D_{5 / 2}-\left({ }^{1} S\right)^{2} P_{3 / 2}^{0}$ \\
\hline 1753.562 & 20 & 57026.78 & -0.013 & $\left({ }^{3} F\right)^{4} F_{3: 2}-\left({ }^{3} P\right)^{2} S_{1: 2}^{0}$ & 1275.937 & 2 & 78373.77 & -0.003 & ${ }^{2} D 1_{3 / 2}-\left({ }^{3} F\right)^{2} D_{5 ; 2}^{0}$ \\
\hline 1752.375 & 200 & 57065.41 & -0.009 & $\left({ }^{3} F\right)^{2} F_{5 / 2}-\left({ }^{1} D\right)^{2} D_{3 / 2}^{0}$ & 1270.936 & 10 & 78682.16 & -0.006 & ${ }^{2} D 1_{5,2}-\left({ }^{3} F\right)^{2} D_{5 ; 2}^{0}$ \\
\hline 1750.368 & 500 & 57130.84 & 0.001 & $\left({ }^{1} G\right)^{2} G_{7 ; 2}-\left({ }^{1} G\right)^{2} F_{5 / 2}^{0}$ & 1270.771 & 1 & 78692.38 & -0.004 & ${ }^{2} D 1_{3: 2}-\left({ }^{3} P\right)^{2} S_{1 / 2}^{0}$ \\
\hline 1742.877 & 20 & 57376.39 & -0.008 & $\left({ }^{3} F\right)^{4} F_{5: 2}-\left({ }^{3} F\right)^{2} G_{7 / 2}^{0}$ & 1269.850 & 200 & 78749.45 & -0.016 & $\left({ }^{1} D\right)^{2} D_{3,2}-\left({ }^{1} S\right)^{2} P_{3,2}^{0}$ \\
\hline 1733.366 & 100 & 57691.22 & -0.005 & $\left({ }^{1} D\right)^{2} D_{3 / 2}-\left({ }^{3} P\right)^{2} D_{3 / 2}^{0}$ & 1263.304 & 1 & 79157.51 & 0.006 & ${ }^{2} D 1_{5}-\left({ }^{3} F\right)^{4} D^{0}$ \\
\hline 1726.017 & 5 & 57936.85 & -0.010 & $\left({ }^{3} F\right)^{4} F_{7 / 2}-\left({ }^{3} F\right)^{2} F_{9,2}^{0}$ & $\begin{array}{l}1203.304 \\
1186.049\end{array}$ & 200 & 84313.54 & 0.018 & $\left.{ }^{2} 1_{5,2}-(F){ }^{3} F\right)^{4} G_{5 i 2}^{0}$ \\
\hline 1724.043 & 10 & 58003.19 & -0.002 & $\left({ }^{3} F\right)^{2} F_{5 ; 2}-\left({ }^{3} P\right)^{4} P_{3 ; 2}^{0}$ & 1181.865 & 1 & 84612.03 & $\begin{array}{r}0.010 \\
-0.002\end{array}$ & ${ }^{2} D \mathrm{l}_{5,2}-\left({ }^{1} D\right)^{2} F_{5,2}^{0}$ \\
\hline 1717.905 & 100 & 58210.43 & -0.004 & $\left({ }^{1} D\right)^{2} D_{3 / 2}-\left({ }^{3} P\right)^{2} D_{5 / 2}^{0}$ & 1162.450 & 5 & 86025.20 & 0.001 & ${ }^{2} D 1_{5: 2}-\left({ }^{1} D\right)^{2} F_{7: 2}^{0}$ \\
\hline 1707.915 & 200 & 58550.92 & -0.005 & $\left({ }^{3} F\right)^{2} F_{5 ; 2}-\left({ }^{1} G\right)^{2} G_{7,2}^{0}$ & 1155.375 & 10 & 86551.98 & 0.007 & ${ }^{2} F_{7: 2}-\left({ }^{3} F\right)^{4} G_{7: 2}^{0}$ \\
\hline 1700.933 & 100 & 58791.26 & -0.007 & $\left({ }^{3} F\right)^{2} F_{7 / 2}-\left({ }^{1} G\right)^{2} H_{9,2}^{0}$ & 1153.939 & 1 & 86659.69 & -0.001 & $\left({ }^{3} F\right)^{4} F_{3 / 2}^{0}$ \\
\hline 1689.265 & 20 & 59197.34 & -0.002 & $\left({ }^{3} F\right)^{4} F_{5: 2}-\left({ }^{3} P\right)^{4} S_{3: 2}^{0}$ & 1149.263 & 5 & 87012.28 & 0.005 & ${ }^{2} D 1_{3: 2}-\left({ }^{t} D\right)^{2} D_{3: 2}^{0}$ \\
\hline 1687.437 & 10 & 59261.47 & -0.001 & $\left({ }^{1} D\right)^{2} D_{5 ; 2}-\left({ }^{3} P\right)^{2} P_{3: 2}^{0}$ & & & & & \\
\hline 1684.828 & 20 & 59353.24 & $-0.00 \mathrm{I}$ & $\left({ }^{1} D\right)^{2} D_{3: 2}-\left({ }^{3} P\right)^{2} P_{12}^{0}$ & 1138.636 & 5 & 87824.37 & 0.006 & 2 \\
\hline 1681.696 & 20 & 59463.77 & -0.004 & $\left({ }^{3} P\right)^{4} P_{5,2}-\left({ }^{3} P\right)^{2} D_{32}^{6}$ & 1115.973 & 5 & 89607.90 & -0.001 & $3 ; 2$ \\
\hline & 100 & 59537.09 & -0.003 & $\left({ }^{3} P\right)^{4} P_{3,2}-\left({ }^{3} P\right)^{2} D_{3 / 2}^{0}$ & 1103.004 & 1 & 90661.50 & 0.003 & $F_{7 / 2}^{0}$ \\
\hline & & & & & 1099.201 & 200 & 90975.17 & $-0.00 \mathrm{I}$ & \\
\hline
\end{tabular}

Table I. Continued$$
\text { Wavelength }
$$
$\left({ }^{3} F\right)^{2} F_{712}-\left({ }^{3} P\right)^{2} D_{5}^{0}$ $\left({ }^{3} F\right)^{4} F_{912}-(D)^{2} F_{72}^{0}$ $\left({ }^{3} P\right)^{4} P_{32}-\left(^{3} P\right)^{2} P^{0}$ $-0.003 \quad\left({ }^{3} F\right)^{4} F_{3,2}-\left({ }^{3} P\right)^{4} D_{1 / 2}^{0}$ $\left({ }^{3} F\right)^{4} F_{52}-\left({ }^{3} P\right)^{4} D_{32}^{0}$

$\begin{array}{lllll}1615.186 & 20 & 61912.37 & 0.005 & \left({ }^{3} F\right)^{4} F_{3: 2}-\left({ }^{1} D\right)^{2} P_{3: 2}^{0}\end{array}$ $1610.788 \quad 200 \quad 62081.41 \quad-0.004 \quad\left({ }^{3} F\right)^{4} F_{7 / 2}-\left({ }^{3} P\right)^{4} D_{5,2}^{0}$ $1606.711 \quad 50 \quad 62238.94$ $1562.312-100-64007.70$

67146.00

70170.37

70904.93

1378.751

1287.64

1285.748

1270.936

1263.304

1162.450 
Table I. Continued

\begin{tabular}{|c|c|c|c|c|c|c|c|c|c|}
\hline $\begin{array}{l}\text { Wavelength } \\
(\AA)\end{array}$ & Int. & $\begin{array}{l}\text { Wavenumber } \\
\left(\mathrm{cm}^{-1}\right)\end{array}$ & $O-C(\AA)$ & $\begin{array}{l}\text { Classification } \\
4 d^{3}, 4 d^{2}() 5 s-4 d^{2}() 5 p\end{array}$ & $\begin{array}{l}\text { Wavelength } \\
(\AA)\end{array}$ & Int. & $\begin{array}{l}\text { Wavenumber } \\
\left(\mathrm{cm}^{-1}\right)\end{array}$ & $O-C(\AA)$ & $\begin{array}{l}\text { Classification } \\
4 d^{3}, 4 d^{2}() 5 s-4 d^{2}() 5 p\end{array}$ \\
\hline 1093.909 & 10 & 91483.77 & -0.004 & ${ }^{2} F_{5 / 2}-\left({ }^{3} F\right)^{4} D_{5 / 2}^{0}$ & 974.710 & 100 & 102594.61 & 0.000 & ${ }^{4} P_{3 / 2}-\left({ }^{3} F\right)^{4} F_{5 / 2}^{0}$ \\
\hline 1089.364 & 10 & 91796.68 & 0.001 & ${ }^{2} F_{7 / 2}-\left({ }^{3} F\right)^{4} D_{5 / 2}^{0}$ & 974.390 & 50 & 102628.31 & 0.000 & ${ }^{2} F_{5 / 2}-\left({ }^{1} G\right)^{2} G_{7 / 2}^{0}$ \\
\hline 1083.330 & 10 & 92307.97 & -0.001 & ${ }^{2} D 2_{3 / 2}-\left({ }^{3} F\right)^{4} G_{5 / 2}^{0}$ & 971.424 & 50 & 102941.66 & 0.001 & ${ }^{2} F_{7,2}-\left({ }^{1} G\right)^{2} G_{7 / 2}^{0}$ \\
\hline 1081.441 & 20 & 92469.21 & 0.001 & ${ }^{2} D l_{3 / 2}-\left({ }^{3} P\right)^{2} D_{3 / 2}^{0}$ & 970.808 & 200 & 103006.98 & 0.002 & ${ }^{2} H_{9 / 2}-\left({ }^{3} F\right)^{2} G_{7 / 2}^{0}$ \\
\hline 1079.843 & 5 & 92606.05 & -0.001 & ${ }^{2} D 2_{5 / 2}-\left({ }^{3} F\right)^{4} G_{5 / 2}^{0}$ & 970.756 & 50 & 103012.49 & 0.000 & ${ }^{4} P_{S / 2}-\left({ }^{3} F\right)^{4} F_{7 / 2}^{0}$ \\
\hline 1077.849 & 50 & 92777.37 & 0.002 & ${ }^{2} D 1_{5 / 2}-\left({ }^{3} P\right)^{2} D_{3 / 2}^{0}$ & 970.456 & 2 & 103044.34 & -0.004 & ${ }^{2} G_{7 / 2}-\left({ }^{3} F\right)^{4} F_{7 / 2}^{0}$ \\
\hline 1077.389 & 20 & 92816.98 & 0.001 & ${ }^{2} F_{7 / 2}-\left({ }^{3} F\right)^{2} D_{5 / 2}^{0}$ & 966.851 & 100 & 103428.55 & -0.001 & ${ }^{2} P_{3 / 2}-\left({ }^{3} F\right)^{2} D_{5 / 2}^{0}$ \\
\hline 1075.401 & 20 & 92988.56 & 0.000 & ${ }^{2} D 1_{3 / 2}^{7 / 2}-\left({ }^{3} P\right)^{2} D_{5 / 2}^{0}$ & 966.638 & 200 & 103451.34 & 0.000 & ${ }^{2} G_{9 / 2}-\left({ }^{3} F\right)^{2} F_{7 / 2}^{0}$ \\
\hline 1071.885 & 20 & 93293.59 & -0.005 & ${ }^{2} F_{7 / 2}-\left({ }^{3} F\right)^{4} D_{7 / 2}^{0}$ & 965.487 & 200 & 103574.67 & 0.002 & ${ }^{2} P_{1 / 2}-\left({ }^{3} P\right)^{2} S_{1 / 2}^{0}$ \\
\hline 1071.852 & 100 & 93296.46 & 0.004 & ${ }^{2} D 1_{5 / 2}-\left({ }^{3} P\right)^{2} D_{5 / 2}^{0}$ & 964.782 & 20 & 103650.35 & 0.007 & ${ }^{2} G_{9 / 2}-\left({ }^{3} F\right)^{4} F_{9 / 2}^{0}$ \\
\hline 1064.940 & 50 & 93902.00 & -0.001 & ${ }^{2} F_{5 / 2}-\left({ }^{3} F\right)^{2} G_{7 / 2}^{0}$ & 964.748 & 50 & 103654.01 & 0.004 & $\left.{ }^{4} P_{3 / 2}-{ }^{3} F\right)^{2} F_{5 / 2}^{0}$ \\
\hline 1062.343 & 100 & 94131.55 & -0.001 & ${ }^{2} D 1_{3 / 2}-\left({ }^{3} P\right)^{2} P_{1 / 2}^{0}$ & 963.885 & 200 & 103746.81 & 0.002 & ${ }^{2} P_{3 / 2}^{3 / 2}-\left({ }^{3} P\right)^{2} S_{1 ; 2}^{0}$ \\
\hline 1057.859 & 20 & 94530.55 & -0.004 & ${ }^{2} D 2_{5 / 2}-\left({ }^{3} F\right)^{4} G_{7 / 2}^{0}$ & 961.404 & 50 & 104014.54 & -0.004 & ${ }^{2} D 2_{s / 2}-\left({ }^{3} P\right)^{4} S_{3 / 2}^{0}$ \\
\hline 1056.489 & 1 & 94653.13 & -0.005 & ${ }^{2} D 2_{3,2}-\left({ }^{3} F\right)^{4} F_{3 / 2}^{0}$ & 960.520 & 100 & 104110.27 & 0.004 & ${ }^{2} F_{7 / 2}-\left({ }^{3} P\right)^{4} P_{5 / 2}^{0}$ \\
\hline 1052.495 & 50 & 95012.32 & -0.004 & ${ }^{2} D 1_{3 / 2}-\left({ }^{3} P\right)^{2} P_{3 / 2}^{0}$ & 960.147 & 200 & 104150.71 & -0.005 & ${ }^{4} P_{5 / 2}-\left({ }^{3} F\right)^{2} F_{7 / 2}^{0}$ \\
\hline 1049.982 & 50 & 95239.72 & -0.001 & ${ }^{2} P_{3 / 2}-\left({ }^{3} F\right)^{4} G_{5 / 2}^{0}$ & 958.887 & 300 & 104287.57 & 0.000 & ${ }^{2} H_{11 / 2}-\left({ }^{3} F\right)^{2} G_{9 / 2}^{0}$ \\
\hline 1049.096 & 100 & 95320.16 & 0.001 & ${ }^{2} D 1_{5 / 2}-\left({ }^{3} P\right)^{2} P_{3 / 2}^{0}$ & 958.130 & 50 & 104369.97 & 0.003 & ${ }^{4} P_{1,2}-\left({ }^{3} F\right)^{2} D_{3 / 2}^{0}$ \\
\hline 1043.642 & 1 & 95818.29 & -0.004 & ${ }^{2} D 2_{3 / 2}^{5 / 2}-\left({ }^{3} F\right)^{4} F_{5 / 2}^{0}$ & 958.058 & 50 & 104377.81 & -0.002 & ${ }^{4} P_{3 / 2}-\left({ }^{3} F\right)^{2} D_{3 / 2}^{0}$ \\
\hline 1043.206 & 50 & 95858.34 & -0.004 & ${ }^{2} F_{7 / 2}-\left({ }^{3} F\right)^{2} G_{9 / 2}^{0}$ & 955.568 & 100 & 104649.80 & 0.001 & ${ }^{2} H_{9 / 2}-\left({ }^{3} F\right)^{2} G_{9 / 2}^{0}$ \\
\hline 1041.352 & 100 & 96029.00 & -0.003 & ${ }^{2} D 1_{s / 2}-\left({ }^{1} G\right)^{2} F_{7 / 2}^{6}$ & 952.633 & 100 & 104972.21 & -0.004 & ${ }^{4} P_{5 / 2}-\left({ }^{3} F\right)^{4} D_{5 / 2}^{0}$ \\
\hline 1040.406 & 50 & 96116.32 & -0.004 & ${ }^{2} D 2_{5 / 2}-\left({ }^{3} F\right)^{4} F_{5 / 2}^{0}$ & 952.348 & 100 & 105003.63 & -0.003 & ${ }^{2} G_{7 / 2}-\left({ }^{3} F\right)^{4} D_{5 / 2}^{0}$ \\
\hline 1030.018 & 10 & 97085.68 & -0.001 & ${ }^{2} H_{11 / 2}-\left({ }^{3} F\right)^{4} G_{9 / 2}^{0}$ & 949.109 & 20 & 105361.97 & 0.003 & ${ }^{2} F_{7 / 2}-\left({ }^{1} G\right)^{2} H_{9 / 2}^{0}$ \\
\hline 1029.926 & 100 & 97094.35 & -0.004 & ${ }^{2} D 1_{3 / 2}-\left({ }^{1} G\right)^{2} F_{5 / 2}^{0}$ & 948.304 & 100 & 105451.41 & 0.000 & ${ }^{4} P_{1 / 2}-\left({ }^{3} F\right)^{4} D_{1 / 2}^{0}$ \\
\hline 1029.060 & 50 & 97176.06 & -0.004 & ${ }^{2} D 2_{s / 2}-\left({ }^{3} F\right)^{2} F_{s / 2}^{0}$ & 948.244 & 10 & 105458.08 & 0.006 & ${ }^{4} P_{3 / 2}-\left({ }^{3} F\right)^{4} D_{1 / 2}^{0}$ \\
\hline 1026.671 & 20 & 97402.18 & 0.000 & ${ }^{2} D l_{s / 2}-\left({ }^{1} G\right)^{2} F_{5 / 2}^{0}$ & 945.456 & 10 & 105769.06 & 0.003 & ${ }^{2} G_{9 / 2}-\left({ }^{3} F\right)^{4} D_{7 / 2}^{0}$ \\
\hline 1026.558 & 20 & 97412.90 & -0.006 & ${ }^{2} P_{1 / 2}-\left({ }^{3} F\right)^{4} F_{3 / 2}^{0}$ & 943.463 & 50 & 105992.49 & -0.004 & ${ }^{4} P_{5 / 2}-\left({ }^{3} F\right)^{2} D_{5 / 2}^{0}$ \\
\hline 1024.740 & 100 & 97585.72 & -0.014 & ${ }^{2} P_{3 / 2}-\left({ }^{3} F\right)^{4} F_{3 / 2}^{0}$ & 943.403 & 100 & 105999.23 & 0.003 & ${ }^{2} D 2_{5 / 2}-\left({ }^{1} D\right)^{2} P_{3 / 2}^{0}$ \\
\hline 1024.576 & 20 & 97601.34 & -0.005 & ${ }^{2} D 2_{3 / 2}-\left({ }^{3} F\right)^{2} D_{3 / 2}^{6}$ & 943.187 & 50 & 106023.51 & 0.000 & ${ }^{2} G_{7 / 2}-\left({ }^{3} F\right)^{2} D_{5 / 2}^{0}$ \\
\hline 1022.465 & 2 & 97802.85 & 0.004 & ${ }^{4} P_{5 / 2}-\left({ }^{3} F\right)^{4} G_{5 / 2}^{0}$ & 941.194 & 50 & 106248.02 & 0.000 & ${ }^{4} P_{1 / 2}-\left({ }^{3} F\right)^{4} D_{3 / 2}^{0}$ \\
\hline 1022.336 & 1 & 97815.20 & 0.000 & ${ }^{2} D 2_{5 / 2}-\left({ }^{3} F\right)^{4} F_{7 / 2}^{0}$ & 941.143 & 100 & 106253.77 & -0.005 & ${ }^{4} P_{3 / 2}-\left({ }^{3} F\right){ }^{4} D_{5 / 2}^{0}$ \\
\hline 1022.127 & 50 & 97835.20 & -0.005 & ${ }^{2} G_{7 / 2}-\left({ }^{3} F\right)^{4} G_{5 / 2}^{0}$ & 939.617 & 50 & 106426.34 & 0.004 & ${ }^{2} D_{3 / 2}-\left({ }^{\prime} D\right)^{2} F_{5 / 2}^{0}$ \\
\hline 1015.908 & 100 & 98434.11 & -0.003 & ${ }^{2} F_{5 / 2}-\left({ }^{1} D\right)^{2} F_{5 / 2}^{0}$ & 939.242 & 200 & 106468.83 & -0.006 & ${ }^{4} P_{5 / 2}-\left({ }^{3} F\right)^{4} D_{7 / 2}^{0}$ \\
\hline 1013.891 & 10 & 98629.93 & -0.006 & ${ }^{2} H_{9 / 2}-\left({ }^{3} F\right)^{4} F_{7 / 2}^{0}$ & 938.092 & 200 & 106599.35 & 0.001 & ${ }^{2} F_{5 / 2}-\left({ }^{3} P\right)^{2} D_{3 / 2}^{0}$ \\
\hline 1012.659 & 100 & 98749.92 & -0.003 & ${ }^{2} P_{3 / 2}-\left({ }^{3} F\right)^{4} F_{5 / 2}^{0}$ & 937.881 & 100 & 106623.33 & 0.002 & ${ }^{2} D 2_{5 / 2}-\left({ }^{3} P\right)^{4} D_{3 / 2}^{0}$ \\
\hline 1010.574 & 200 & 98953.66 & -0.008 & ${ }^{2} D 2_{5 / 2}-\left({ }^{3} F\right)^{2} F_{7 / 2}^{0}$ & 937.668 & 20 & 106647.55 & 0.003 & ${ }^{2} P_{3 / 2}-\left({ }^{3} P\right)^{4} S_{3 / 2}^{0}$ \\
\hline 1009.797 & 50 & 99029.80 & -0.011 & ${ }^{2} G_{9 / 2}-\left({ }^{3} F\right)^{4} G_{7 / 2}^{0}$ & 937.285 & 200 & 106691.13 & 0.003 & ${ }^{2} G_{9 / 2}-\left({ }^{3} F\right)^{2} G_{7 / 2}^{0}$ \\
\hline 1009.225 & 5 & 99085.93 & -0.013 & ${ }^{4} P_{3 / 2}-\left({ }^{3} F\right)^{4} G_{S / 2}^{0}$ & 936.993 & 50 & 106724.38 & 0.004 & ${ }^{2} D 2_{5 / 2}-\left({ }^{\prime} D\right)^{2} F_{5 / 2}^{0}$ \\
\hline 1005.259 & 200 & 99476.85 & -0.004 & ${ }^{2} D 2_{3 / 2}-\left({ }^{3} F\right)^{4} D_{5 / 2}^{0}$ & 933.548 & 10 & 107118.22 & 0.004 & ${ }^{2} F_{5,2}-\left({ }^{3} P\right)^{2} D_{5,2}^{0}$ \\
\hline 1003.965 & 100 & 99605.06 & -0.006 & ${ }^{2} H_{11 / 2}\left({ }^{3} F\right)^{4} F_{9 / 2}^{0}$ & 932.200 & 10 & 107273.11 & 0.003 & ${ }^{4} P_{3 / 2}-\left({ }^{3} F\right)^{2} D_{3 / 2}^{0}$ \\
\hline 1003.106 & 10 & 99690.36 & -0.010 & ${ }^{2} F_{5 / 2}-\left({ }^{3} P\right)^{4} D_{5 / 2}^{0}$ & 931.185 & 5 & 107390.04 & 0.003 & ${ }^{4} P_{5 / 2}-\left({ }^{3} F\right)^{2} G_{7 / 2}^{0}$ \\
\hline 1002.413 & 1 & 99759.28 & -0.003 & ${ }^{2} G_{7 / 2}-\left({ }^{3} F\right)^{4} G_{7 / 2}^{0}$ & 930.911 & 300 & 107421.65 & 0.001 & ${ }^{2} G_{7 / 2}-\left({ }^{3} F\right)^{2} G_{7 / 2}^{0}$ \\
\hline 1002.326 & 5 & 99767.94 & -0.009 & ${ }^{2} H_{9 / 2}-\left({ }^{3} F\right)^{2} F_{7 / 2}^{0}$ & 930.822 & 300 & 107431.92 & 0.001 & ${ }^{2} F_{7 / 2}-\left({ }^{3} P\right)^{2} D_{5 / 2}^{0}$ \\
\hline 1002.245 & 20 & 99776.00 & 0.007 & ${ }^{2} D 2_{5 / 2}-\left({ }^{3} F\right)^{4} D_{3 / 2}^{0}$ & 929.493 & 10 & 107585.53 & -0.008 & ${ }^{4} P_{1 / 2}-\left({ }^{3} P\right)^{2} S_{1 / 2}^{0}$ \\
\hline 1001.903 & 200 & 99810.06 & -0.007 & ${ }^{2} P_{3 / 2}-\left({ }^{3} F\right)^{2} F_{5 / 2}^{0}$ & 929.437 & 100 & 107592.01 & 0.000 & ${ }^{4} P_{3 / 2}^{1 / 2}-\left({ }^{3} P\right)^{2} S_{1 / 2}^{0}$ \\
\hline 1001.521 & 10 & 99848.13 & -0.010 & ${ }^{2} F_{5 / 2}-\left({ }^{1} D\right)^{2} F_{5 / 2}^{0}$ & 929.072 & 200 & 107634.28 & 0.000 & ${ }^{2} D 2_{3 / 2}-\left({ }^{1} D\right)^{2} P_{1 / 2}^{0}$ \\
\hline 1000.325 & 3 & 99967.51 & -0.007 & ${ }^{2} H_{9 / 2}-\left({ }^{3} F\right)^{4} F_{9 / 2}^{0}$ & 928.895 & 2 & 107654.79 & 0.006 & ${ }^{4} F_{7 / 2}-\left({ }^{3} F\right)^{4} G_{5 / 2}^{0}$ \\
\hline 998.391 & 100 & 100161.15 & -0.007 & ${ }^{2} F_{7 / 2}-\left({ }^{1} D\right)^{2} F_{7 / 2}^{0}$ & 926.099 & 10 & 107979.81 & 0.005 & ${ }^{2} D 2_{5 / 2}-\left({ }^{3} P\right)^{4} D_{5 / 2}^{0}$ \\
\hline 996.400 & 200 & 100361.30 & -0.008 & ${ }^{2} P_{1 / 2}\left({ }^{3} F\right)^{2} D_{3 / 2}^{0}$ & 924.748 & 5 & 108137.56 & 0.006 & ${ }^{2} D 2_{5 / 2}-\left({ }^{1} D\right)^{2} F_{7 / 2}^{0}$ \\
\hline 995.050 & 200 & 100497.46 & -0.007 & ${ }^{2} D 2_{3,2}-\left({ }^{3} F\right)^{2} D_{5 / 2}^{0}$ & 923.072 & 300 & 108333.91 & 0.003 & ${ }^{2} G_{9 / 2}-\left({ }^{3} F\right)^{2} G_{9 / 2}^{0}$ \\
\hline 994.695 & 100 & 100533.32 & -0.007 & ${ }^{2} P_{3 / 2}^{3 / 2}-\left({ }^{3} F\right)^{2} D_{3 / 2}^{0}$ & 921.997 & 20 & 108460.22 & 0.008 & ${ }^{2} P_{1 / 2}-\left({ }^{1} D\right)^{2} P_{3 / 2}^{0}$ \\
\hline 992.106 & 200 & 100795.68 & -0.009 & ${ }^{2} D 2_{5 / 2}-\left({ }^{3} F\right)^{2} D_{5 / 2}^{0}$ & 921.874 & 10 & 108474.69 & 0.002 & ${ }^{4} F_{9 / 2}-\left({ }^{3} F\right)^{4} G_{7 / 2}^{0}$ \\
\hline 991.907 & 100 & 100815.90 & -0.006 & ${ }^{2} D 2_{3 / 2}-\left({ }^{3} P\right)^{2} S_{1 / 2}^{0}$ & 920.529 & 200 & 108633.18 & 0.000 & ${ }^{2} P_{3 / 2}-\left({ }^{1} D\right)^{2} P_{3 / 2}^{0}$ \\
\hline 998.789 & 20 & 101.132 .89 & -0.007 & ${ }^{2} G_{9 / 2}-\left({ }^{3} F\right)^{4} G_{9 / 2}^{0}$ & 917.837 & 10 & 108951.80 & 0.005 & ${ }^{2} H_{9 / 2}-\left({ }^{1} D\right)^{2} F_{7 / 2}^{0}$ \\
\hline 998.693 & 5 & 101143.63 & -0.008 & ${ }^{2} F_{5 / 2}-\left({ }^{1} D\right)^{2} D_{3 / 2}^{0}$ & 916.891 & 50 & 109064.21 & 0.002 & ${ }^{2} G_{7 / 2}-\left({ }^{3} F\right)^{2} G_{9 / 2}^{0}$ \\
\hline 987.444 & 200 & 101271.56 & -0.007 & ${ }^{2} D 2_{5 / 2}-\left({ }^{3} F\right)^{4} D_{7 / 2}^{0}$ & 916.295 & 200 & 109135.15 & 0.006 & ${ }^{2} D 2_{3 / 2}-(D)^{2} D_{3 / 2}^{0}$ \\
\hline 987.011 & 100 & 101315.99 & -0.001 & ${ }^{2} F_{5 / 2}-\left({ }^{3} P\right)^{4} D_{7 / 2}^{0}$ & 916.239 & 100 & 109141.82 & 0.002 & ${ }^{2} F_{5 / 2}-\left({ }^{3} P\right)^{2} P_{3 / 2}^{0}$ \\
\hline 986.722 & 10 & 101345.66 & -0.010 & ${ }^{2} G_{7 / 2}-\left({ }^{3} F\right)^{4} F_{5 / 2}^{0}$ & 915.655 & 200 & 109211.43 & 0.000 & ${ }^{4} P_{5 / 2}-\left({ }^{3} P\right)^{4} S_{3 / 2}^{0}$ \\
\hline 985.900 & 20 & 101430.16 & -0.007 & ${ }^{4} P_{3 / 2}-\left({ }^{3} F\right)^{4} F_{3 / 2}^{0}$ & 915.277 & 50 & 109256.54 & 0.006 & ${ }^{2} P_{3 / 2}-\left({ }^{3} P\right)^{4} D_{3 / 2}^{0}$ \\
\hline 981.065 & 100 & 101930.04 & -0.004 & ${ }^{2} F_{7 / 2}-\left({ }^{1} D\right)^{2} D_{5 / 2}^{0}$ & 913.948 & 200 & 109415.41 & -0.002 & ${ }^{4} F_{3 / 2}-\left({ }^{3} F\right)^{4} G_{5 / 2}^{0}$ \\
\hline 979.605 & 2 & 102081.96 & -0.011 & ${ }^{2} F_{S / 2}-\left({ }^{3} P\right)^{4} P_{3 / 2}^{0}$ & 913.797 & 200 & 109433.49 & 0.003 & $\left.{ }^{2} D 2_{5 / 2}-{ }^{\mathrm{l}} D\right)^{2} D_{3 / 2}^{0}$ \\
\hline 979.570 & 10 & 102085.60 & -0.005 & ${ }^{2} H_{9 / 2}-\left({ }^{3} F\right)^{4} D_{7 / 2}^{0}$ & 912.580 & 2 & 109579.43 & 0.003 & ${ }^{4} F_{7 / 2}-\left({ }^{3} F\right)^{4} G_{7 / 2}^{0}$ \\
\hline 978.353 & 100 & 102212.59 & -0.005 & ${ }^{2} F_{7 / 2}-\left({ }^{1} G\right)^{2} G_{9 / 2}^{0}$ & 912.353 & 10 & 109606.69 & 0.003 & ${ }^{2} D 2_{5 / 2}-\left({ }^{3} P\right)^{4} D_{7 / 2}^{0}$ \\
\hline 978.104 & 100 & 102238.61 & -0.003 & ${ }^{2} P_{1 / 2}-\left({ }^{3} F\right)^{4} D_{3 / 2}^{0}$ & 911.975 & 100 & 109652.12 & 0.008 & ${ }^{2} D 2_{3 / 2}-\left({ }^{3} P\right)^{4} P_{1 / 2}^{0}$ \\
\hline 976.822 & 2 & 102372.79 & 0.002 & ${ }^{4} P_{5 / 2}-\left({ }^{3} F\right)^{2} F_{5 / 2}^{0}$ & 910.330 & 5 & 109850.27 & 0.003 & ${ }^{2} F_{5 / 2}-\left({ }^{1} G\right)^{2} F_{7 / 2}^{0}$ \\
\hline 976.520 & 100 & 102404.45 & 0.000 & ${ }^{2} G_{7 / 2}-\left({ }^{3} F\right)^{2} F_{S / 2}^{0}$ & 909.861 & 200 & 109906.89 & 0.004 & ${ }^{2} D 2_{5 / 2}-\left({ }^{l} D\right)^{2} D_{5 / 2}^{0}$ \\
\hline 976.466 & 100 & 102410.12 & 0.002 & ${ }^{2} P_{3 / 2}-\left({ }^{3} F\right)^{4} D_{3 / 2}^{0}$ & 908.486 & 50 & 110073.24 & 0.005 & ${ }^{2} D 2_{3 / 2}-\left({ }^{3} P\right)^{4} P_{3 / 2}^{0}$ \\
\hline
\end{tabular}

Table I. Continued

0.00

963.885

961.404

960.520

958.887

958.130

952.633

952.348

948.244

945.456

943.187

939.617

938.092

37.881

936.993

932.200

931.185

929.493

923.072

921.874

916.891

916.295

915.277

913.948

912.580

911.975

909.861

908.486

$50 \quad 110073.24$

0.005 
Table I. Continued

\begin{tabular}{|c|c|c|c|c|c|c|c|c|c|}
\hline $\begin{array}{l}\text { Wavelength } \\
(\AA)\end{array}$ & Int. & $\begin{array}{l}\text { Wavenumber } \\
\left(\mathrm{cm}^{-1}\right)\end{array}$ & $O-C(\AA)$ & $\begin{array}{l}\text { Classification } \\
4 d^{3}, 4 d^{2}() 5 s-4 d^{2}() 5 p\end{array}$ & $\begin{array}{l}\text { Wavelength } \\
(\AA)\end{array}$ & Int. & $\begin{array}{l}\text { Wavenumber } \\
\left(\mathrm{cm}^{-1}\right)\end{array}$ & $O-C(\AA)$ & $\begin{array}{l}\text { Classification } \\
4 d^{3}, 4 d^{2}() 5 s-4 d^{2}() 5 p\end{array}$ \\
\hline 907.745 & 200 & 110163.09 & 0.008 & ${ }^{2} F_{7 ; 2}-\left({ }^{1} G\right)^{2} F^{07: 2}$ & 871.677 & 50 & 114721.39 & 0.006 & ${ }^{2} P_{3 / 2}-\left({ }^{3} P\right)^{4} P_{5 / 2}^{0}$ \\
\hline 906.034 & 20 & 110371.13 & 0.006 & ${ }^{2} D 2_{5: 2}-\left({ }^{3} P\right)^{4} P_{5 / 2}^{0}$ & 871.052 & 100 & 114803.70 & 0.005 & ${ }^{4} P_{5 / 2}-\left({ }^{3} P\right)^{4} D_{7 / 2}^{0}$ \\
\hline 905.853 & 100 & 110393.18 & 0.006 & ${ }^{2} P_{1 / 2}-\left({ }^{1} D\right)^{2} P_{1 / 2}^{0}$ & 870.899 & 200 & 114823.87 & 0.001 & ${ }^{4} F_{7 / 2}-\left({ }^{3} F\right)^{4} D_{5 ; 2}^{0}$ \\
\hline 905.630 & 200 & 110420.37 & 0.007 & ${ }^{2} H_{9,2}-\left({ }^{3} P\right)^{4} D_{7 / 2}^{0}$ & 870.813 & 100 & 114835.21 & 0.004 & ${ }^{2} G_{7 / 2}-\left({ }^{3} P\right)^{4} D_{7 / 2}^{0}$ \\
\hline 905.102 & 200 & 110484.78 & 0.006 & ${ }^{4} P_{1: 2}-\left({ }^{3} P\right)^{4} S_{3: 2}^{0}$ & 870.394 & 10 & 114890.49 & 0.000 & ${ }^{2} D 2_{5 / 2}-\left({ }^{3} P\right)^{2} D_{3 / 2}^{0}$ \\
\hline 905.038 & 200 & 110492.59 & 0.002 & ${ }^{4} P_{3 / 2}-\left({ }^{3} P\right)^{4} S_{3 / 2}^{0}$ & 868.774 & 100 & 115104.73 & $-0.00 \mathrm{l}$ & ${ }^{4} P_{5,2}-\left({ }^{1} D\right)^{2} D_{5: 2}^{0}$ \\
\hline 904.487 & 300 & 110559.90 & -0.003 & ${ }^{4} F_{3 / 2}-\left({ }^{3} F\right)^{4} G_{7 / 2}^{0}$ & 868.539 & 50 & 115135.87 & $0.00 \mathrm{I}$ & ${ }^{2} G_{7 / 2}-\left({ }^{1} D\right)^{2} D_{5 / 2}^{0}$ \\
\hline 904.337 & 100 & 110578.24 & 0.002 & ${ }^{4} F_{9 / 2}-\left({ }^{3} F\right){ }^{4} G_{9 / 2}^{0}$ & 867,941 & 300 & 115215.20 & 0.003 & ${ }^{4} F_{9 / 2}-\left({ }^{3} F\right)^{4} D D_{7 / 2}^{0}$ \\
\hline 904.047 & 50 & 110613.71 & 0.003 & ${ }^{2} P_{3 / 2}-\left({ }^{3} P\right)^{4} D_{5 / 2}^{0}$ & 866.418 & 100 & 115417.73 & 0.002 & $\left.{ }^{2} G_{9 ; 2}-\left({ }^{1} G\right)^{2} G_{7,2}^{0}\right\}$ \\
\hline 903.821 & 300 & 110641.37 & 0.002 & ${ }^{2} H_{11 ; 2}-\left({ }^{1} G\right)^{2} G_{9 / 2}^{0}$ & & & & 0.006 & $\left.{ }^{2} G_{7 / 2}-\left({ }^{1} G\right)^{2} G_{9 / 2}^{0}\right\}$ \\
\hline 902.220 & 200 & 110837.71 & 0.008 & ${ }^{2} D 1_{3 / 2}-\left({ }^{1} S\right)^{2} P_{1 / 2}^{0}$ & 865.284 & 100 & 115568.99 & 0.002 & ${ }^{4} P_{5 / 2}-\left({ }^{3} P\right)^{4} P_{3 / 2}^{0}$ \\
\hline 901.559 & 10 & 110918.97 & 0.004 & ${ }^{2} D 2_{s / 2}-(G)^{2} G_{7 / 2}^{0}$ & 864.647 & 300 & 115654.13 & 0.006 & ${ }^{2} H_{11 / 2}-\left({ }^{\prime} G\right)^{2} H_{11 / 2}^{0}$ \\
\hline 900.873 & 100 & 111003.43 & 0.004 & ${ }^{2} H_{9 / 2}-\left({ }^{1} G\right)^{2} G_{9 ; 2}^{0}$ & 863.636 & 200 & 115789.52 & -0.001 & ${ }^{4} F_{3 / 2}-\left({ }^{3} F\right)^{4} D_{1 / 2}^{0}$ \\
\hline 899.563 & 10 & 111165.08 & 0.003 & ${ }^{4} F_{7 / 2}-\left({ }^{3} F\right)^{4} F_{5 / 2}^{0}$ & 863.515 & 200 & 115805.74 & 0.002 & ${ }^{4} F_{5 / 2}-\left({ }^{3} F\right)^{4} D_{3 / 2}^{0}$ \\
\hline 899.306 & 100 & 111196.85 & 0.000 & ${ }^{4} P_{5 / 2}-\left({ }^{1} D\right)^{2} P_{3 / 2}^{0}$ & 863.228 & 200 & 115844.25 & 0.001 & ${ }^{4} F_{7,2}-\left({ }^{3} F\right)^{2} D_{5 ;: 2}^{0}$ \\
\hline 899.093 & 200 & 111223.19 & 0.007 & ${ }^{2} F_{5 / 2}-\left({ }^{1} G\right)^{2} F_{5: 2}^{0}$ & 862.775 & 5 & 115905.07 & 0.001 & ${ }^{4} P_{1,2}-\left({ }^{1} D\right)^{2} D_{3,2}^{0}$ \\
\hline 895.391 & 200 & 111683.05 & 0.002 & ${ }^{4} F_{7,2}-\left({ }^{3} F\right)^{4} G_{9 / 2}^{0}$ & 861.203 & 5 & 116116.64 & 0.001 & ${ }^{4} P_{5 / 2}-\left({ }^{1} G\right)^{2} G_{7 / 2}^{0}$ \\
\hline 894.992 & 200 & 111732.84 & 0.007 & ${ }^{2} H_{9 / 2}-\left({ }^{1} G\right)^{2} G_{7 / 2}^{0}$ & 861.054 & 50 & 116136.73 & 0.008 & ${ }^{4} F_{9 / 2}-\left({ }^{3} F\right)^{2} G_{7 / 2}^{0}$ \\
\hline 894.774 & 200 & 111760.06 & $\left\{\begin{array}{l}-0.001 \\
-0.001\end{array}\right.$ & $\left.\begin{array}{l}{ }^{4} F_{9 / 2}-\left({ }^{3} F\right)^{4} F_{7 / 2}^{0} \\
{ }^{4} F_{3 / 2}-\left({ }^{3} F\right)^{4} F_{3 / 2}^{0}\end{array}\right\}$ & $\begin{array}{l}860.187 \\
859.695\end{array}$ & $\begin{array}{l}50 \\
50\end{array}$ & $\begin{array}{l}116253.79 \\
116320.32\end{array}$ & $\begin{array}{l}0.006 \\
0.001\end{array}$ & $\begin{array}{l}{ }^{2} D 2_{3 / 2}-\left({ }^{3} P\right)^{2} P_{1 / 2}^{0} \\
{ }^{4} F_{7 / 2}-\left({ }^{3} F\right)^{4} D_{7 / 2}^{0}\end{array}$ \\
\hline 894.537 & 5 & 111789.67 & 0.006 & ${ }^{2} D 2_{3 / 2}-\left({ }^{3} P\right)^{4} P_{5 / 2}^{0}$ & 859.211 & 20 & 116385.84 & 0.001 & ${ }^{4} P_{3 / 2}-\left({ }^{1} D\right)^{2} D_{5 ; 2}^{0}$ \\
\hline 894.289 & 30 & 111820.67 & 0.001 & ${ }^{4} P_{5 / 2}-\left({ }^{3} P\right)^{4} D_{3 / 2}^{0}$ & 858.937 & 10 & 116422.97 & -0.004 & ${ }^{4} P_{1 / 2}-\left({ }^{3} P\right)^{4} P_{1 / 2}^{0}$ \\
\hline 893.234 & 200 & 111952.74 & 0.007 & ${ }^{2} G_{7 / 2}-\left({ }^{1} D\right)^{2} F_{5 / 2}^{0}$ & 858.886 & 100 & 116429.88 & 0.000 & ${ }^{4} P_{3 / 2}-\left({ }^{3} P\right)^{4} P_{1 / 2}^{0}$ \\
\hline 892.211 & 50 & 112081.11 & 0.001 & ${ }^{4} P_{1,2}-\left({ }^{3} P\right)^{4} D_{1 / 2}^{0}$ & 857.734 & 100 & 116586.26 & -0.002 & ${ }^{4} F_{3 / 2}-\left({ }^{3} F\right)^{4} D_{3 / 2}^{0}$ \\
\hline 892.154 & 100 & 112088.27 & 0.002 & $\left.{ }^{2} D 2_{5: 2}-\left({ }^{3} P\right)^{4} P_{5 ; 2}^{0}\right\}$ & 855.984 & 100 & 116824.61 & -0.004 & ${ }^{4} F_{5,2}-\left({ }^{3} F\right)^{2} D_{5 ;, 2}^{0}$ \\
\hline & & & 0.003 & $\left.{ }^{4} P_{3,2}-\left({ }^{3} P\right)^{4} D_{1 / 2}^{0}\right\}$ & 855.849 & 100 & 116843.04 & 0.001 & ${ }^{4} P_{1 / 2}-\left({ }^{3} P\right)^{4} P_{3 / 2}^{0}$ \\
\hline 891.704 & 200 & 112144.83 & 0.003 & ${ }^{4} F_{S / 2}-\left({ }^{3} F\right)^{4} F_{5 / 2}^{0}$ & 855.795 & 5 & 116850.41 & 0.001 & ${ }^{4} P_{3 / 2}-\left({ }^{3} P\right)^{4} P_{3 / 2}^{0}$ \\
\hline 891.065 & 100 & 112225.25 & 0.000 & ${ }^{4} F_{7 / 2}-\left({ }^{3} F\right)^{2} F_{3 / 2}^{0}$ & 853.718 & 100 & 117134.69 & 0.003 & ${ }^{2} D 2_{3 ; 2}-\left({ }^{3} P\right)^{2} P_{3 / 2}^{0}$ \\
\hline 889.066 & 50 & 112477.58 & 0.005 & ${ }^{4} P_{3,2}^{7 / 2}-\left({ }^{1} D\right)^{2} P_{3 / 2}^{0}$ & 852.938 & 2 & 117241.81 & 0.005 & ${ }^{4} F_{7 / 2}-\left({ }^{3} F\right)^{2} G_{7 / 2}^{0}$ \\
\hline 888.571 & 5 & 112540.24 & 0.006 & ${ }^{2} P_{3 ; 2}-\left({ }^{1} D\right)^{2} D_{5,2}^{6}$ & 852.620 & 200 & 117285.54 & 0.002 & ${ }^{4} P_{5 / 2}-\left({ }^{3} P\right)^{4} P_{9 / 2}^{0}$ \\
\hline 888.225 & 20 & 112584.08 & 0.006 & ${ }^{2} P_{3,2}^{3 / 2}-\left({ }^{3} P\right)^{4} P_{5 / 2}^{0}$ & 852.392 & 2 & 117316.91 & 0.002 & ${ }^{2} G_{7 / 2}-\left({ }^{3} P\right)^{4} P_{5 / 2}^{0}$ \\
\hline 887.813 & 300 & 112636.33 & 0.003 & $\left.{ }^{2} G_{9,2}-{ }^{(} D\right)^{2} F_{7,2}^{0}$ & 851.549 & 100 & 117433.05 & 0.001 & ${ }^{2} D 2_{5 / 2}-\left({ }^{3} P\right)^{2} P_{3 / 2}^{0}$ \\
\hline 886.269 & 10 & 112832.56 & 0.007 & ${ }^{2} P_{1 / 2}-\left({ }^{3} P\right)^{4} P_{3 / 2}^{0}$ & 850.312 & 3 & 117603.89 & 0.001 & ${ }^{4} F_{3 / 2}-\left({ }^{3} F\right)^{2} D_{5 / 2}^{0}$ \\
\hline 886.015 & 400 & 112864.90 & -0.001 & ${ }^{4} F_{7 / 2}-\left({ }^{3} F\right)^{4} F_{7 / 2}^{0}$ & 849.040 & 50 & 117780.08 & 0.003 & ${ }^{4} F_{9 / 2}-\left({ }^{3} F\right)^{2} G_{9 / 2}^{0}$ \\
\hline 885.760 & 200 & 112897.39 & 0.001 & ${ }^{4} F_{9 / 2}-\left({ }^{3} F\right)^{2} F_{7 / 2}^{0}$ & 846.443 & 8 & 118141.44 & 0.002 & ${ }^{2} D 2_{s / 2}-(G)^{2} F_{7 i 2}^{0}$ \\
\hline 885.540 & 100 & 112925.44 & -0.002 & ${ }^{4} F_{3,2}-\left({ }^{3} F\right)^{4} F_{7 / 2}^{0}$ & 845.865 & 2 & 118222.17 & 0.001 & ${ }^{4} F_{5 ; 2}-\left({ }^{3} F\right)^{2} G_{7 / 2}^{0}$ \\
\hline 884.919 & 10 & 113004.69 & 0.007 & ${ }^{2} P_{3 / 2}-\left({ }^{3} P\right)^{4} P_{3 / 2}^{0,2}$ & 843.404 & 20 & 118567.14 & 0.000 & ${ }^{4} P_{3 / 2}-\left({ }^{3} P\right)^{4} P_{5 / 2}^{0}$ \\
\hline 884.830 & 200 & 113016.06 & 0.004 & ${ }^{4} F_{9 ; 2}-\left({ }^{3} F\right)^{4} G_{11 / 2}^{0}$ & 841.145 & 10 & 118885.56 & -0.002 & ${ }^{4} F_{7 / 2}-\left({ }^{3} F\right)^{2} G_{9,2}^{0}$ \\
\hline 884.195 & 500 & 113097.22 & 0.001 & ${ }^{4} F_{9 ; 2}-\left({ }^{3} F\right)^{4} F_{9 / 2}^{0}$ & 840.651 & 20 & 118955.42 & 0.004 & ${ }^{2} H_{9 ; 2}-\left({ }^{\mathrm{l}} G\right)^{2} F_{7 / 2}^{0}$ \\
\hline 883.564 & 5 & 113177.99 & -0.002 & ${ }^{4} P_{5,2}-\left({ }^{3} P\right)^{4} D_{5,2}^{0}$ & 840.233 & 10 & 119014.60 & -0.002 & ${ }^{2} P_{1 / 2}-\left({ }^{3} P\right)^{2} P_{1 / 2}^{0}$ \\
\hline 883.361 & 200 & 113204.00 & $\{-0.003$ & $\left.{ }^{4} P_{3 / 2}^{5 / 2}-\left({ }^{1} D\right)^{2} F_{5 / 2}^{0}\right\}$ & 839.024 & 50 & 119186.10 & 0.002 & ${ }^{2} P_{3 / 2}-\left({ }^{3} P\right)^{2} P_{1 / 2}^{0}$ \\
\hline & & & 0.007 & $\left.{ }^{4} F_{5 / 2}^{3 / 2}-\left({ }^{3} F\right)^{2} F_{5 / 2}^{0}\right\}$ & 838.809 & 5 & 119216.65 & 0.003 & ${ }^{2} D 2_{3 ; 2}-\left({ }^{1} G\right)^{2} F_{5 / 2}^{0}$ \\
\hline 883.321 & 200 & 113209.12 & 0.000 & ${ }^{2} G_{7 / 2}^{5 / 2}-\left({ }^{3} P\right)^{4} D_{5 / 2}^{0}$ & 835.410 & 50 & 119701.70 & -0.001 & ${ }^{2} G_{9,2}-\left({ }^{1} G\right)^{2} H_{11,2}^{0}$ \\
\hline 882.338 & so & 113335.25 & 0.002 & ${ }^{4} P_{5: 2}-\left({ }^{1} D\right)^{2} F_{7 / 2}^{0}$ & 821.189 & 1 & 121774.64 & -0.001 & ${ }^{4} F_{7 / 2}-\left({ }^{1} D\right)^{2} F_{5 / 2}^{0}$ \\
\hline 882.093 & 100 & 113366.73 & 0.002 & ${ }^{2} G_{72}-\left({ }^{1} D\right)^{2} F_{7,2}^{0}$ & 819.471 & 2 & 122029.94 & -0.008 & ${ }^{4} F_{5: 2}-\left({ }^{1} D\right)^{2} P_{3,2}^{0}$ \\
\hline 880.853 & 20 & 113526.32 & 0.003 & ${ }^{2} D 1_{3 ; 2}-\left({ }^{1} S\right)^{2} P_{3,2}^{0}$ & 819.110 & 5 & 122083.72 & -0.005 & ${ }^{4} F_{9 / 2}-\left({ }^{1} D\right)^{2} F_{7 / 2}^{0}$ \\
\hline 878.801 & 100 & 113791.40 & 0.003 & ${ }^{2} H_{3,2}-\left({ }^{1} G\right)^{2} H_{9,2}^{3 / 2}$ & 816.856 & 20 & 122420.59 & -0.009 & ${ }^{4} F_{3 / 2}-\left({ }^{3} P\right)^{4} D_{1 / 2}^{0}$ \\
\hline 878.474 & 100 & 113833.76 & 0.009 & ${ }^{2} D 1_{5,2}-\left({ }^{1} S\right)^{2} P^{0}{ }^{0,2}$ & 815.386 & 1 & 122641.30 & -0.007 & ${ }^{2} G_{9 / 2}-\left({ }^{1} G\right)^{2} F_{7 / 2}^{0}$ \\
\hline 878.394 & 100 & 113844.12 & 0.003 & ${ }^{4} F_{5: 2}-\left({ }^{3} F\right)^{4} F_{7 / 2}^{0}$ & 815.305 & 50 & 122653.48 & -0.005 & ${ }^{4} F_{5 / 2}-\left({ }^{3} P\right)^{4} D_{3 / 2}^{0}$ \\
\hline 877.750 & 200 & 113927.65 & 0.004 & ${ }^{4} F_{5: 2}-\left({ }^{3} F\right)^{2} D_{3: 2}^{0}$ & 812.801 & 100 & 123031.34 & -0.009 & ${ }^{4} F_{7 / 2}-\left({ }^{3} P\right)^{4} D_{5 / 2}^{0}$ \\
\hline 877.308 & 10 & 113985.05 & -0.001 & ${ }^{4} F_{3}-\left({ }^{3} F\right)^{2} F_{5 / 2}^{0,2}$ & & & & 0.000 & $\left.{ }^{4} P_{3 / 2}-\left({ }^{3} P\right)^{2} P_{1 / 2}^{0}\right\}$ \\
\hline 876.384 & 100 & 114105,23 & 0.002 & ${ }^{2} G_{9 / 2}-\left({ }^{3} P\right)^{4} D_{7 / 2}^{6 / 2}$ & 810.152 & 2 & 123433.62 & -0.006 & ${ }^{4} F_{3 / 2}-\left({ }^{3} P\right)^{4} D_{3 / 2}^{0}$ \\
\hline 876.013 & 200 & $114\lfloor 53.55$ & 0.005 & ${ }^{2} H_{9,2}-\left({ }^{\prime} G\right)^{2} H_{9,2}^{0}$ & 809.369 & 100 & 123533.03 & -0.008 & ${ }^{4} F_{9 / 2}-\left({ }^{3} P\right)^{4} D_{7 / 2}^{9}$ \\
\hline 875.642 & 50 & 114201.92 & 0.002 & ${ }^{4} F_{7 / 2}-\left({ }^{3} F\right)^{4} F_{9 / 2}^{0}$ & 806.387 & 10 & 124009.93 & -0.001 & ${ }^{4} F_{5 / 2}-\left({ }^{3} P\right)^{4} D_{5 / 2}^{0}$ \\
\hline 874.047 & 10 & 114410.32 & 0.005 & ${ }^{4} P_{3,}-\left({ }^{1} D\right)^{2} P_{1}^{0}$ & 805.362 & 20 & 124167.76 & -0.002 & ${ }^{4} F_{5 ; 2}-\left({ }^{1} D\right)^{2} F_{7 ; 2}^{0}$ \\
\hline 873.676 & 100 & 114458.90 & 0.002 & $\begin{array}{l}\left.{ }^{4} P_{3,2}-(D)\right)^{2} P_{1 / 2}^{0} \\
{ }^{4} P_{3,2}-\left({ }^{3} P\right)^{4} D_{5 ; 2}^{0}\end{array}$ & 802.200 & 2 & 124657.19 & -0.004 & ${ }^{4} F_{7 / 2}-\left({ }^{3} P\right)^{4} D_{7 / 2}^{0}$ \\
\hline 872.362 & 20 & 114631.31 & -0.001 & ${ }^{4} P_{5,2}-\left({ }^{1} D\right)^{2} D_{3,2}^{0,2}$ & 801.639 & 2 & 124744.43 & -0.004 & ${ }^{2} G_{7 / 2}-\left({ }^{1} G\right)^{2} F_{5 / 2}^{0}$ \\
\hline 871.933 & 100 & 114687.71 & 0.004 & 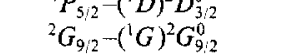 & 800.858 & 2 & 124866.08 & -0.012 & ${ }^{4} F_{9 / 2}-\left({ }^{1} G\right)^{2} G_{7 / 2}^{0}$ \\
\hline 871.777 & 50 & 114708.23 & 0.000 & $\begin{array}{l}G_{9 / 2}-(G)^{2} G_{9,2} \\
{ }^{4} F_{3,2}-\left({ }^{3} F\right)^{2} D_{3,0}^{0}\end{array}$ & 800.266 & 1 & 124958.45 & -0.010 & ${ }^{4} F_{7 ; 2}-\left({ }^{1} D\right)^{2} D_{5 ; 2}^{0}$ \\
\hline
\end{tabular}

Table I. Continued 
Table II. Even levels of triply ionized molybdenum (MoIV). The level, level uncertainty and $O-C$ values are given in units of $\mathrm{cm}^{-1}$. The number of transitions to each level is given in the column headed "No". The "Leading Percentages" are given in LS coupling

\begin{tabular}{|c|c|c|c|c|c|c|c|c|c|}
\hline Config. & Term & $J$ & Level & Unc. & No. & $O-C$ & \multicolumn{3}{|c|}{ Leading percentages } \\
\hline $4 d^{3}$ & ${ }^{4} F$ & $\begin{array}{l}3 / 2 \\
5 / 2 \\
7 / 2 \\
9 / 2\end{array}$ & $\begin{array}{r}0.00 \\
778.02 \\
1759.76 \\
2864.58\end{array}$ & $\begin{array}{l}0.25 \\
0.26 \\
0.22 \\
0.27\end{array}$ & $\begin{array}{l}10 \\
12 \\
16 \\
12\end{array}$ & $\begin{array}{r}-10 \\
-10 \\
-10 \\
0\end{array}$ & $\begin{array}{r}99 \\
100 \\
100 \\
98\end{array}$ & & \\
\hline $4 d^{3}$ & ${ }^{4} P$ & $\begin{array}{l}1 / 2 \\
3 / 2 \\
5 / 2\end{array}$ & $\begin{array}{l}10337.77 \\
10330.59 \\
11611.99\end{array}$ & $\begin{array}{l}0.30 \\
0.19 \\
0.16\end{array}$ & $\begin{array}{l}10 \\
20 \\
19\end{array}$ & $\begin{array}{r}0 \\
20 \\
20\end{array}$ & $\begin{array}{l}93 \\
83 \\
99\end{array}$ & $14^{2} P$ & \\
\hline $4 d^{3}$ & ${ }^{2} G$ & $\begin{array}{l}7 / 2 \\
9 / 2\end{array}$ & $\begin{array}{l}11580.50 \\
12310.80\end{array}$ & $\begin{array}{l}0.17 \\
0.24\end{array}$ & $\begin{array}{l}17 \\
13\end{array}$ & $\begin{array}{r}-20 \\
0\end{array}$ & $\begin{array}{l}99 \\
83\end{array}$ & $15^{2} H$ & \\
\hline $4 d^{3}$ & ${ }^{2} P$ & $\begin{array}{l}3 / 2 \\
1 / 2\end{array}$ & $\begin{array}{l}14175.62 \\
14347.72\end{array}$ & $\begin{array}{l}0.21 \\
0.30\end{array}$ & $\begin{array}{r}17 \\
8\end{array}$ & $\begin{array}{l}20 \\
60\end{array}$ & $\begin{array}{l}45 \\
93\end{array}$ & $31^{2} D 2$ & $15^{4} P$ \\
\hline $4 d^{3}$ & ${ }^{2} H$ & $\begin{array}{r}9 / 2 \\
11 / 2\end{array}$ & $\begin{array}{l}15995.13 \\
16357.48\end{array}$ & $\begin{array}{l}0.26 \\
0.29\end{array}$ & $\begin{array}{r}12 \\
6\end{array}$ & $\begin{array}{l}20 \\
30\end{array}$ & $\begin{array}{r}85 \\
100\end{array}$ & $15^{2} G$ & \\
\hline $4 d^{3}$ & ${ }^{2} D 2$ & $\begin{array}{l}5 / 2 \\
3 / 2\end{array}$ & $\begin{array}{l}16809.32 \\
17107.35\end{array}$ & $\begin{array}{l}0.15 \\
0.19\end{array}$ & $\begin{array}{l}24 \\
16\end{array}$ & $\begin{array}{r}-60 \\
0\end{array}$ & $\begin{array}{l}81 \\
43\end{array}$ & $\begin{array}{l}17^{2} D 1 \\
41^{2} P\end{array}$ & $13^{2} D 1$ \\
\hline $4 d^{3}$ & ${ }^{2} F$ & $\begin{array}{l}7 / 2 \\
5 / 2\end{array}$ & $\begin{array}{l}24787.03 \\
25100.40\end{array}$ & $\begin{array}{l}0.17 \\
0.20\end{array}$ & $\begin{array}{l}14 \\
19\end{array}$ & $\begin{array}{l}-30 \\
-30\end{array}$ & $\begin{array}{l}99 \\
99\end{array}$ & & \\
\hline $4 d^{3}$ & ${ }^{2} D 1$ & $\begin{array}{l}5 / 2 \\
3 / 2\end{array}$ & $\begin{array}{l}38922.29 \\
39230.53\end{array}$ & $\begin{array}{l}0.14 \\
0.12\end{array}$ & $\begin{array}{l}12 \\
15\end{array}$ & $\begin{array}{r}30 \\
-20\end{array}$ & $\begin{array}{l}81 \\
77\end{array}$ & $\begin{array}{l}16^{2} D 2 \\
23^{2} D 2\end{array}$ & \\
\hline $4 d^{2}\left({ }^{3} F\right) 5 s$ & ${ }^{4} F$ & $\begin{array}{l}3 / 2 \\
5 / 2 \\
7 / 2 \\
9 / 2\end{array}$ & $\begin{array}{l}60896.28 \\
61626.19 \\
62708.50 \\
64046.46\end{array}$ & $\begin{array}{l}0.07 \\
0.05 \\
0.06 \\
0.07\end{array}$ & $\begin{array}{l}17 \\
21 \\
18 \\
12\end{array}$ & $\begin{array}{r}20 \\
10 \\
10 \\
0\end{array}$ & $\begin{array}{r}99 \\
98 \\
99 \\
100\end{array}$ & & \\
\hline $4 d^{2}\left({ }^{3} F\right) 5 s$ & ${ }^{2} F$ & $\begin{array}{l}5 / 2 \\
7 / 2\end{array}$ & $\begin{array}{l}69178.07 \\
71358.31\end{array}$ & $\begin{array}{l}0.05 \\
0.05\end{array}$ & $\begin{array}{l}25 \\
20\end{array}$ & $\begin{array}{l}-30 \\
-10\end{array}$ & $\begin{array}{l}94 \\
98\end{array}$ & & \\
\hline $4 d^{2}\left({ }^{3} P\right) 5 s$ & ${ }^{4} P$ & $\begin{array}{l}1 / 2 \\
3 / 2 \\
5 / 2\end{array}$ & $\begin{array}{l}71750.36 \\
72162.82 \\
72236.20\end{array}$ & $\begin{array}{l}0.06 \\
0.05 \\
0.06\end{array}$ & $\begin{array}{l}12 \\
17 \\
16\end{array}$ & $\begin{array}{l}-10 \\
-10 \\
-30\end{array}$ & $\begin{array}{r}100 \\
93 \\
70\end{array}$ & $27\left({ }^{1} D\right) 2 D$ & \\
\hline $4 d^{2}\left({ }^{1} D\right) 5 s$ & ${ }^{2} D$ & $\begin{array}{l}3 / 2 \\
5 / 2\end{array}$ & $\begin{array}{l}74008.77 \\
74981.08\end{array}$ & $\begin{array}{l}0.04 \\
0.04\end{array}$ & $\begin{array}{l}24 \\
17\end{array}$ & $\begin{array}{r}10 \\
-10\end{array}$ & $\begin{array}{l}89 \\
68\end{array}$ & $\begin{array}{r}7\left({ }^{3} P\right) 4 P \\
29\left({ }^{3} P\right) 4 P\end{array}$ & \\
\hline $4 d^{2}\left({ }^{1} G\right) 5 s$ & ${ }^{2} G$ & $\begin{array}{l}9 / 2 \\
7 / 2\end{array}$ & $\begin{array}{l}79106.26 \\
79193.56\end{array}$ & $\begin{array}{l}0.05 \\
0.05\end{array}$ & $\begin{array}{r}9 \\
10\end{array}$ & $\begin{array}{r}-20 \\
20\end{array}$ & $\begin{array}{r}100 \\
99\end{array}$ & & \\
\hline $4 d^{2}\left({ }^{3} P\right) 5 s$ & ${ }^{2} P$ & $\begin{array}{l}1 / 2 \\
3 / 2\end{array}$ & $\begin{array}{l}79898.80 \\
81052.21\end{array}$ & $\begin{array}{l}0.05 \\
0.05\end{array}$ & $\begin{array}{r}9 \\
13\end{array}$ & $\begin{array}{l}20 \\
20\end{array}$ & $\begin{array}{c}99 \\
96\end{array}$ & & \\
\hline $4 d^{2}\left({ }^{1} S\right) 5 s$ & ${ }^{2} S$ & $1 / 2$ & 99783.80 & 0.08 & 3 & 0 & 99 & & \\
\hline
\end{tabular}

Table III. Odd levels of triply ionized molybdenum (MoIV). The level, level uncertainty and $O-C$ values are given in units of $\mathrm{cm}^{-1}$. The number of transitions from each level is given in the column headed "No"

\begin{tabular}{|c|c|c|c|c|c|c|c|c|c|}
\hline \multirow{2}{*}{$\frac{\text { Config. }}{4 d^{2}\left({ }^{3} F\right) 5 p}$} & \multirow{2}{*}{$\begin{array}{l}\text { Term } \\
{ }^{4} G\end{array}$} & \multirow{2}{*}{$\begin{array}{r}J \\
5 / 2 \\
7 / 2 \\
9 / 2 \\
11 / 2\end{array}$} & \multirow{2}{*}{$\begin{array}{l}\text { Level } \\
109415.25 \\
111339.53 \\
113443.02 \\
115881.12\end{array}$} & \multirow{2}{*}{$\begin{array}{l}\text { Unc. } \\
0.06 \\
0.06 \\
0.07 \\
0.15\end{array}$} & \multirow{2}{*}{$\begin{array}{l}\text { No. } \\
14 \\
12 \\
7 \\
2\end{array}$} & \multirow{2}{*}{$\begin{array}{l}O-C \\
-140 \\
-100 \\
-80 \\
-60\end{array}$} & \multicolumn{3}{|c|}{ Leading Percentages } \\
\hline & & & & & & & \multicolumn{3}{|c|}{$\begin{array}{rl}82 & 12\left({ }^{3} F\right)^{2} F \\
90 & \\
92 & \\
100 & \end{array}$} \\
\hline $4 d^{2}\left({ }^{3} F\right) 5 p$ & ${ }^{4} F$ & $\begin{array}{l}3 / 2 \\
5 / 2 \\
7 / 2 \\
9 / 2\end{array}$ & $\begin{array}{l}111760.03 \\
112925.24 \\
114624.49 \\
115961.91\end{array}$ & $\begin{array}{l}0.05 \\
0.05 \\
0.09 \\
0.07\end{array}$ & $\begin{array}{r}12 \\
16 \\
11 \\
9\end{array}$ & $\begin{array}{r}110 \\
0 \\
90 \\
100\end{array}$ & $\begin{array}{l}75 \\
73 \\
90 \\
82\end{array}$ & $\begin{array}{l}19\left({ }^{3} F\right)^{2} D \\
12\left({ }^{3} F\right)^{2} D \\
8\left({ }^{3} F\right)^{4} G\end{array}$ & $7\left({ }^{3} F\right)^{4} D$ \\
\hline $4 d^{2}\left({ }^{3} F\right) 5 p$ & ${ }^{2} F$ & $\begin{array}{l}5 / 2 \\
7 / 2\end{array}$ & $\begin{array}{l}113984.98 \\
115762.16\end{array}$ & $\begin{array}{l}0.05 \\
0.05\end{array}$ & $\begin{array}{l}16 \\
15\end{array}$ & $\begin{array}{l}-120 \\
-110\end{array}$ & $\begin{array}{l}41 \\
61\end{array}$ & $\begin{array}{l}19\left({ }^{3} F\right)^{4} F \\
25\left({ }^{3} F\right)^{4} D\end{array}$ & $\begin{array}{r}16\left({ }^{3} F\right)^{4} G \\
6\left({ }^{3} F\right)^{4} G\end{array}$ \\
\hline $4 d^{2}\left({ }^{3} F\right) 5 p$ & ${ }^{2} D$ & $\begin{array}{l}3 / 2 \\
5 / 2\end{array}$ & $\begin{array}{l}114708.24 \\
117604.08\end{array}$ & $\begin{array}{l}0.07 \\
0.05\end{array}$ & $\begin{array}{l}16 \\
20\end{array}$ & $\begin{array}{r}-60 \\
-120\end{array}$ & $\begin{array}{c}42 \\
32\end{array}$ & $\begin{array}{l}23\left({ }^{3} F\right)^{4} F \\
26\left({ }^{3} F\right)^{4} D\end{array}$ & $\begin{array}{l}17\left({ }^{3} F\right)^{4} D \\
18\left({ }^{3} P\right)^{2} D\end{array}$ \\
\hline $4 d^{2}\left({ }^{3} F\right) 5 p$ & ${ }^{4} D$ & $\begin{array}{l}1 / 2 \\
3 / 2 \\
5 / 2 \\
7 / 2\end{array}$ & $\begin{array}{l}115789.36 \\
116586.00 \\
116583.82 \\
118080.17\end{array}$ & $\begin{array}{l}0.11 \\
0.09 \\
0.06 \\
0.09\end{array}$ & $\begin{array}{r}5 \\
12 \\
15 \\
13\end{array}$ & $\begin{array}{r}100 \\
0 \\
-70 \\
-60\end{array}$ & $\begin{array}{l}79 \\
60 \\
47 \\
55\end{array}$ & $\begin{array}{l}17\left({ }^{3} P\right)^{4} D \\
17\left({ }^{3} P\right)^{4} D \\
19\left({ }^{3} F\right)^{2} F \\
19\left({ }^{3} F\right)^{2} F\end{array}$ & $\begin{array}{l}13\left({ }^{3} F\right)^{2} D \\
13\left({ }^{3} F\right)^{2} D \\
10\left({ }^{3} P\right)^{4} D\end{array}$ \\
\hline
\end{tabular}


Table III. Continued

\begin{tabular}{|c|c|c|c|c|c|c|c|c|c|}
\hline Config. & Term & $J$ & Level & Unc. & No. & $O-C$ & \multicolumn{3}{|c|}{ Leading Percentages } \\
\hline $4 d^{2}\left({ }^{3} P\right) 5 p$ & ${ }^{2} S$ & $1 / 2$ & 117922.63 & 0.08 & 10 & -40 & 92 & & \\
\hline $4 d^{2}\left({ }^{3} F\right) 5 p$ & ${ }^{2} G$ & $\begin{array}{l}7 / 2 \\
9 / 2\end{array}$ & $\begin{array}{l}119002.33 \\
120645.02\end{array}$ & $\begin{array}{l}0.10 \\
0.10\end{array}$ & $\begin{array}{l}14 \\
11\end{array}$ & $\begin{array}{l}210 \\
150\end{array}$ & $\begin{array}{l}78 \\
73\end{array}$ & $\begin{array}{l}12\left({ }^{l} G\right)^{2} G \\
14\left({ }^{l} G\right)^{2} G\end{array}$ & $11\left({ }^{3} F\right)^{4} F$ \\
\hline $4 d^{2}\left({ }^{3} P\right) 5 p$ & ${ }^{4} S$ & $3 / 2$ & 120823.46 & 0.08 & 11 & -240 & 65 & $25\left(^{(} D\right)^{2} P$ & \\
\hline $4 d^{2}\left({ }^{3} P\right) 5 p$ & ${ }^{4} D$ & $\begin{array}{l}1 / 2 \\
3 / 2 \\
5 / 2 \\
7 / 2\end{array}$ & $\begin{array}{l}122419.26 \\
123432.84 \\
124789.75 \\
126416.33\end{array}$ & $\begin{array}{l}0.08 \\
0.08 \\
0.06 \\
0.09\end{array}$ & $\begin{array}{r}7 \\
14 \\
16 \\
16\end{array}$ & $\begin{array}{r}60 \\
-60 \\
110 \\
80\end{array}$ & $\begin{array}{l}80 \\
49 \\
69 \\
54\end{array}$ & $\begin{array}{l}18\left({ }^{3} F\right)^{4} D \\
27\left({ }^{1} D\right)^{2} P \\
15\left({ }^{1} D\right)^{2} F \\
31\left({ }^{1} G\right)^{2} G\end{array}$ & $\begin{array}{r}12\left({ }^{3} P\right)^{4} S \\
10\left({ }^{3} F\right)^{4} D \\
5\left({ }^{3} F\right)^{2} G\end{array}$ \\
\hline $4 d^{2}(' D) 5 p$ & ${ }^{2} P$ & $\begin{array}{l}3 / 2 \\
1 / 2\end{array}$ & $\begin{array}{l}122808.84 \\
124741.62\end{array}$ & $\begin{array}{l}0.12 \\
0.09\end{array}$ & $\begin{array}{r}12 \\
7\end{array}$ & $\begin{array}{r}-90 \\
-230\end{array}$ & $\begin{array}{l}19 \\
79\end{array}$ & $\begin{array}{l}31\left({ }^{3} P\right)^{4} D \\
16\left({ }^{3} P\right)^{4} P\end{array}$ & $19\left({ }^{3} P\right)^{4} S$ \\
\hline $4 d^{2}\left({ }^{1} D\right) 5 p$ & ${ }^{2} F$ & $\begin{array}{l}5 / 2 \\
7 / 2\end{array}$ & $\begin{array}{l}123534.18 \\
124947.54\end{array}$ & $\begin{array}{l}0.06 \\
0.07\end{array}$ & $\begin{array}{l}14 \\
17\end{array}$ & $\begin{array}{l}280 \\
160\end{array}$ & $\begin{array}{l}61 \\
45\end{array}$ & $\begin{array}{l}13\left({ }^{3} F\right)^{2} F \\
18\left({ }^{1} G\right)^{2} G\end{array}$ & $\begin{array}{r}7\left({ }^{3} P\right)^{4} D \\
18\left({ }^{3} P\right)^{4} D\end{array}$ \\
\hline $4 d^{2}\left({ }^{\prime} D\right) 5 p$ & ${ }^{2} D$ & $\begin{array}{l}3 / 2 \\
5 / 2\end{array}$ & $\begin{array}{l}126243.19 \\
126716.64\end{array}$ & $\begin{array}{l}0.11 \\
0.09\end{array}$ & $\begin{array}{l}14 \\
14\end{array}$ & $\begin{array}{r}70 \\
-20\end{array}$ & $\begin{array}{l}40 \\
46\end{array}$ & $\begin{array}{l}21\left({ }^{3} P\right)^{4} P \\
35\left({ }^{3} P\right)^{4} P\end{array}$ & $\begin{array}{r}19\left({ }^{1} D\right)^{2} P \\
7\left({ }^{3} P\right)^{4} D\end{array}$ \\
\hline $4 d^{2}\left({ }^{3} P\right) 5 p$ & ${ }^{4} P$ & $\begin{array}{l}1 / 2 \\
3 / 2 \\
5 / 2\end{array}$ & $\begin{array}{l}126760.46 \\
127181.20 \\
128897.78\end{array}$ & $\begin{array}{l}0.10 \\
0.09 \\
0.07\end{array}$ & $\begin{array}{r}8 \\
16 \\
13\end{array}$ & $\begin{array}{r}180 \\
150 \\
-30\end{array}$ & $\begin{array}{l}79 \\
75 \\
59\end{array}$ & $\begin{array}{l}13\left({ }^{1} D\right)^{2} P \\
19\left({ }^{1} D\right)^{2} D \\
38\left({ }^{1} D\right)^{2} D\end{array}$ & \\
\hline $4 d^{2}\left({ }^{1} G\right) 5 p$ & ${ }^{2} G$ & $\begin{array}{l}7 / 2 \\
9 / 2\end{array}$ & $\begin{array}{l}127728.82 \\
126999.09\end{array}$ & $\begin{array}{l}0.08 \\
0.11\end{array}$ & $\begin{array}{l}13 \\
10\end{array}$ & $\begin{array}{r}110 \\
90\end{array}$ & $\begin{array}{l}35 \\
71\end{array}$ & $\begin{array}{l}33\left({ }^{1} D\right)^{2} F \\
18\left({ }^{3} F\right)^{2} G\end{array}$ & $\begin{array}{l}17\left({ }^{3} P\right)^{4} D \\
11\left({ }^{(} G\right)^{2} H\end{array}$ \\
\hline $4 d^{2}\left({ }^{1} G\right) 5 p$ & ${ }^{2} H$ & $\begin{array}{r}9 / 2 \\
11 / 2\end{array}$ & $\begin{array}{l}130149.35 \\
132012.37\end{array}$ & $\begin{array}{l}0.10 \\
0.15\end{array}$ & $\begin{array}{l}6 \\
3\end{array}$ & $\begin{array}{l}120 \\
110\end{array}$ & $\begin{array}{r}87 \\
100\end{array}$ & $13(G)^{2} G$ & \\
\hline $4 d^{2}\left({ }^{3} P\right) 5 p$ & ${ }^{2} D$ & $\begin{array}{l}3 / 2 \\
5 / 2\end{array}$ & $\begin{array}{l}131699.83 \\
132219.07\end{array}$ & $\begin{array}{l}0.08 \\
0.09\end{array}$ & $\begin{array}{r}12 \\
9\end{array}$ & $\begin{array}{l}-60 \\
-30\end{array}$ & $\begin{array}{l}62 \\
72\end{array}$ & $\begin{array}{l}17\left({ }^{3} P\right)^{2} P \\
19\left(^{3} F\right)^{2} D\end{array}$ & $12\left({ }^{3} F\right)^{2} D$ \\
\hline $4 d^{2}\left(^{3} P 5 p\right.$ & ${ }^{2} p$ & $\begin{array}{l}1 / 2 \\
3 / 2\end{array}$ & $\begin{array}{l}133362.00 \\
134242.50\end{array}$ & $\begin{array}{l}0.08 \\
0.08\end{array}$ & $\begin{array}{l}10 \\
12\end{array}$ & $\begin{array}{r}50 \\
-30\end{array}$ & $\begin{array}{l}94 \\
77\end{array}$ & $11\left({ }^{3} P\right)^{2} D$ & $6\left({ }^{1} D\right)^{2} D$ \\
\hline $4 d^{2}(G) 5 p$ & ${ }^{2} F$ & $\begin{array}{l}7 / 2 \\
5 / 2\end{array}$ & $\begin{array}{l}134951.06 \\
136324.44\end{array}$ & $\begin{array}{l}0.11 \\
0.13\end{array}$ & $\begin{array}{r}13 \\
9\end{array}$ & $\begin{array}{l}-220 \\
-260\end{array}$ & $\begin{array}{l}91 \\
95\end{array}$ & & \\
\hline $4 d^{2}\left({ }^{1} S\right) 5 p$ & ${ }^{2} P$ & $\begin{array}{l}1 / 2 \\
3 / 2\end{array}$ & $\begin{array}{l}150069.22 \\
152757.31\end{array}$ & $\begin{array}{l}0.14 \\
0.19\end{array}$ & $\begin{array}{l}3 \\
6\end{array}$ & $\begin{array}{l}0 \\
0\end{array}$ & $\begin{array}{l}94 \\
97\end{array}$ & & \\
\hline
\end{tabular}

Table IV. LSF and HFR parameter values for the $4 d^{3}, 4 d^{2} 5 s$ and $4 d^{2} 5 p$ configurations of triply ionized molybdenum (Mo IV) in $\mathrm{cm}^{-1}$

\begin{tabular}{llccc}
\hline Config. & Parameter & LSF & HRF & LSF/HRF \\
\hline $4 d^{3}$ & $E_{\mathrm{av}}$ & $14588(10)$ & - & - \\
& $F^{2}(d d)$ & $50411(37)$ & 63051 & 0.80 \\
& $F^{4}(d d)$ & $32830(77)$ & 41431 & 0.79 \\
& $\zeta d$ & $810(7)$ & 796 & 1.01 \\
& $\alpha$ & $38(2)$ & - & - \\
$4 d^{2} 5 s$ & $\beta$ & $-313(31)$ & - & - \\
& $E_{\mathrm{av}}$ & $71732(13)$ & 72375 & 0.99 \\
& $F^{2}(d d)$ & $52632(62)$ & 65612 & 0.80 \\
& $F^{4}(d d)$ & $34979(89)$ & 43294 & 0.81 \\
& $\llbracket d$ & $878(8)$ & 864 & 1.02 \\
& $G^{2}(d s)$ & $12685(64)$ & 15887 & 0.80 \\
& $\alpha$ & $33(2)$ & - & - \\
& $\beta$ & $-339(29)$ & - & - \\
& $R^{2}(d d, d s)$ & $-8830(700)$ & -17415 & 0.51
\end{tabular}

Table IV. Continued

\begin{tabular}{llcrc}
\hline Config. & Parameter & LSF & HRF & LSF/HRF \\
\hline $4 d^{2} 5 p$ & $E_{\text {av }}$ & $122801(23)$ & 122387 & 1.00 \\
& $F^{2}(d d)$ & $52890(250)$ & 66044 & 0.80 \\
& $F^{4}(d d)$ & $35460(310)$ & 43614 & 0.82 \\
& $\llbracket d$ & $926(23)$ & 873 & 1.06 \\
& $\llbracket p$ & $2136(55)$ & 1698 & 1.26 \\
& $F^{2}(d p)$ & $19490(180)$ & 24874 & 0.78 \\
& $G^{1}(d p)$ & $7534(100)$ & 9821 & 0.77 \\
& $G^{3}(d p)$ & $4320(240)$ & 8595 & 0.50 \\
& $\alpha$ & $38(6)$ & - & - \\
& $\beta$ & $-240(100)$ & - & - \\
& \multicolumn{5}{c}{ Standard deviation $=141 \mathrm{~cm}^{-1}$} \\
\end{tabular}

Standard deviation $=30 \mathrm{~cm}^{-1}$ 